\title{
ON THE CHARACTERS OF $p$-SOLVABLE GROUPS
}

\author{
BY \\ P. FONG(1)
}

Introduction. In the theory of group characters, modular representation theory has explained some of the regularities in the behavior of the irreducible characters of a finite group; not unexpectedly, the theory in turn poses new problems of its own. These problems, which are asked by Brauer in [4], seem to lie deep. In this paper we look at the situation for solvable groups. We can answer some of the questions in [4] for these groups, and in doing so, obtain new properties for their characters. Finite solvable groups have recently been the object of much investigation by group theorists, especially with the end of relating the structure of such groups to their Sylow $p$-subgroups. Our work does not lie quite in this direction, although we have one result tying up arithmetic properties of the characters to the structure of certain $p$-subgroups. Since the prime number $p$ is always fixed, we can actually work in the more general class of $p$-solvable groups, and shall do so.

Let $(H)$ be a finite group of order $g=p^{a} g_{0}$, where $p$ is a fixed prime number, $a$ is an integer $\geqq 0$, and $\left(p, g_{0}\right)=1$. In the modular theory, the main results of which are in $[2 ; 3 ; 5 ; 9]$, the characters of the irreducible complex-valued representations of $(\xi)$, or as we shall say, the irreducible characters of $B$, are partitioned into disjoint sets, these sets being the so-called blocks of $\$$ for the prime $p$. Each block $B$ has attached to it a $p$-subgroup $\mathfrak{D}$ of $B$ determined up to conjugates in $\left(\mathfrak{S}\right.$, the defect group of the block $B$. If $\mathfrak{D}$ has order $p^{d}$, in which case we say $B$ has defect $d$, and if $\chi_{\mu}$ is an irreducible character in $B$, then the degree of $\chi_{\mu}$ is divisible by $p$ to the exponent $a-d+e_{\mu}$, where the nonnegative integer $e_{\mu}$ is defined as the height of $\chi_{\mu}$. Now let (S) be a $p$-solvable group, that is, (S) has a composition series such that each factor is either a $p$-group or a $p^{\prime}$-group, a $p^{\prime}$-group being one of order prime to $p$. The following is then true: Let $B$ be a block of $B$ with defect group $\mathfrak{D}$. If $\mathfrak{D}$ is abelian, then every character $\chi_{\mu}$ in $B$ has height 0 . Conversely, if $B$ is the block containing the 1 -character, and if every character in $B$ has height 0 , then $\mathfrak{D}$ is abelian. In particular, this gives a necessary and sufficient condition for the Sylow $p$-subgroups of $B$ to be abelian.

In the general modular theory of finite groups, each irreducible character $\chi_{\mu}$ can be decomposed into a sum of irreducible modular characters $\phi_{\rho}$ of $(S)$,

$$
\chi_{\mu}=\sum d_{\mu \rho} \phi_{\rho}
$$

Received by the editors May 18, 1960.

(1) The author is a National Science Foundation Postdoctoral Fellow. This article includes part of a doctoral thesis submitted to Harvard University in 1959. I wish to express my thanks to Professor Brauer for his kind and helpful advice. 
If $d_{\mu \rho} \neq 0$, we say that $\phi_{\rho}$ is a modular constituent of $\chi_{\mu}$. The non-negative integers $d_{\mu \rho}$ are the decomposition numbers of $\$ \mathcal{S}$, and the sums $c_{\rho \sigma}=\sum_{\mu} d_{\mu \rho} d_{\mu \sigma}$ are the Cartan invariants of ( $\$$. Since an irreducible modular character $\phi_{\rho}$ is in a block $B$ if and only if some irreducible character $\chi_{\mu}$ in $B$ contains $\phi_{\rho}$ as a modular constituent, the matrices $D=\left(d_{\mu_{\rho}}\right)$ and $C=\left(c_{\rho \sigma}\right)$ can be decomposed so as to correspond to the different blocks of $(B$. If $(B)$ is $p$-solvable, the following are also true: Let $B$ be a block of defect $d$. Then the Cartan invariants of $B$ satisfy the inequality $c_{\rho \sigma} \leqq p^{d}$.

In $\$ 1$ we study the relation between the block structure of a group $\$$ and that of a normal subgroup $\mathfrak{S}$ of $\mathfrak{S}$. This is perhaps of independent interest. In $\S 2$ we prove next a reduction step for the case when $\mathfrak{S}$ is a $p^{\prime}$-group. This is the important induction step. Finally, in $\$ 3$ we apply these results to finite $p$-solvable groups and prove the results mentioned above, as well as assorted others for this class of groups.

Notation. (B) is always a finite group of order $g=p^{a} g_{0}$, where $\left(p, g_{0}\right)=1$. $\Omega_{1}, \Omega_{2}, \cdots, \Omega_{k}$ are the conjugate classes of $\mathbb{H}$, and $G_{\alpha}$ a representative of the class $\Re_{\alpha}$. For any subset $\mathfrak{A}$ of $\mathfrak{H}, \mathfrak{N}(\mathfrak{U})$ and $\mathfrak{S}(\mathfrak{A})$ are the normalizer and centralizer of $\mathfrak{A}$ in $\mathfrak{S}$. Set $n(\mathfrak{A})=(\mathfrak{N}(\mathfrak{U}): 1)$. If $\mathfrak{A}$ is a one element set $\{A\}$, we write $\mathfrak{N}(A)$ for $\mathfrak{N}(\mathfrak{U})$. An element $G$ of $\mathscr{S}$ is $p$-regular if its order is prime to $p, p$-singular if its order is divisible by $p$. Let $\nu$ be the exponential valuation of the rational field determined by $p$, normalized so that $\nu(p)=1$. The defect $\nu(G)$ of an element $G$ in $B$ is $\nu(n(G))$; a defect group of $G$ is a Sylow $p$-subgroup of $\mathfrak{R}(G)$.

Let $\Omega$ be a finite algebraic number field containing the $g$ th roots of unity. By [1] the ordinary absolutely irreducible representations of $\&$ can be written with coefficients in $\Omega . \mathfrak{X}_{1}, \mathfrak{X}_{2}, \cdots, \mathfrak{X}_{k}$ will denote the distinct nonequivalent ones, and $\chi_{1}, \chi_{2}, \cdots, \chi_{k}$ their corresponding characters. If $K_{\alpha}$ is the sum of the elements in $\Omega_{\alpha}$ in the group algebra $\Gamma(\xi, \Omega)$ of $B$ over $\Omega$, then the function

$$
\omega_{\mu}\left(K_{\alpha}\right)=\frac{g}{n\left(G_{\alpha}\right)} \frac{\chi_{\mu}\left(G_{\alpha}\right)}{x_{\mu}}, \quad x_{\mu}=\chi_{\mu}(1),
$$

defines a linear character of the center $3(\xi, \Omega)$ of $\Gamma(\xi), \Omega)$. We shall write $\omega_{\mu}\left(G_{\alpha}\right)$ for $\omega_{\mu}\left(K_{\alpha}\right)$.

In $\Omega$ let $\mathfrak{p}$ be a fixed prime divisor of $p$, with $\mathfrak{D}$ as ring of local integers and $\mathfrak{D} / \mathfrak{p}=\Omega^{*}$ as the residue class field. The modular absolutely irreducible representations of $\mathfrak{B S}^{\mathrm{j}}$ can be written in $\Omega^{*}$ (see [1]). $\mathfrak{F}_{1}, \mathfrak{F}_{2}, \cdots, \mathfrak{F}_{m}$ will denote the different nonequivalent ones, and $\phi_{1}, \phi_{2}, \cdots, \phi_{m}$ their corresponding complex-valued characters. Unless the word "modular" is used, a character of $(s)$ is always an ordinary one. The blocks of $\$$ will be denoted by $B_{1}, B_{2}, \cdots, B_{n}$. Each block $B_{\tau}$ corresponds to the primitive idempotent $\eta_{\tau}$ of the center $\left.3(\xi), \Omega^{*}\right)$ of the group algebra of $(\xi)$ over $\Omega^{*}$ and to the linear character $\psi_{\tau}$ of $3\left(\xi, \Omega^{*}\right)$. We shall also write $\psi_{\tau}\left(G_{\alpha}\right)$ for $\psi_{\tau}\left(K_{\alpha}\right)$. Any further notation will be introduced when used. 
1. The block structure of a group with a normal subgroup. We begin this section with some remarks on the action of automorphisms on blocks. Let $\alpha$ be any automorphism of $\mathcal{H}$. $\alpha$ permutes the irreducible characters of $\&$ by

$$
\alpha: \chi_{\mu} \rightarrow \chi_{\mu}^{\alpha} \text {, }
$$

where $\chi_{\mu}^{\alpha}$ is defined by $\chi_{\mu}^{\alpha}(G)=\chi_{\mu}\left(G^{\alpha}\right)$ for all $G$ in (S).

THEOREM (1A). Let $\alpha$ be an automorphism of the finite group (\$) which leaves every character $\chi_{\mu}$ in a block $B_{\tau}$ fixed, that is, $\chi_{\mu}^{\alpha}=\chi_{\mu}$ for all $\chi_{\mu}$ in $B_{\tau}$. If $P$ is an element in a defect group $\mathfrak{D}$ of $B_{\tau}$, then $P$ and $P^{\alpha}$ are conjugate in $(B)$.

Proof. Let the characters $\chi_{\mu}$ in $B_{\tau}$ be indexed by $\mu$ in $S$. There exists a $p$ regular element $V$ in $(S)$ such that $\mathfrak{D}$ is a defect group of $V$ and $\psi_{\tau}(V) \neq 0$. If $P$ and $P^{\alpha}$ were not conjugate in $(S)$, then by $[3],(7 \mathrm{C})$,

$$
\sum_{\mu \text { in } S} \chi_{\mu}\left((P V)^{\alpha}\right) \chi_{\mu}\left((P V)^{-1}\right)=0 .
$$

By assumption $\chi_{\mu}\left((P V)^{\alpha}\right)=\chi_{\mu}(P V)$ for $\mu$ in $S$. Therefore the sum can be rewritten as

$$
\sum_{\mu \text { in } S} \chi_{\mu}(P V) \chi_{\mu}\left((P V)^{-1}\right)=0
$$

The sum being one of non-negative summands, we must have $\chi_{\mu}(P V)=0$ for all $\mu$ in $S$. If $\chi_{\mu}$ has height 0 , this implies that $\chi_{\mu}(V) \equiv \chi_{\mu}(P V)=0(\bmod \mathfrak{p})$, which is impossible. Therefore $P$ and $P^{\alpha}$ are conjugate in $\$$. This completes the proof.

Two characters $\chi_{\lambda}$ and $\chi_{\mu}$ are in the same block of $B$ if and only if

$$
\omega_{\lambda}(G) \equiv \omega_{\mu}(G)(\bmod p)
$$

for all $p$-regular $G$ in (S). It follows immediately from this congruence that $\chi_{\lambda}$ and $\chi_{\mu}$ are in the same block $B_{\tau}$ of $B$ if and only if $\chi_{\lambda}^{\alpha}$ and $\chi_{\mu}^{\alpha}$ are in the same block $B_{\sigma}$ of $B$ for some $\tau$ and $\sigma$. In other words, $\alpha$ permutes the blocks of $\$$.

$$
\alpha: B_{\tau} \rightarrow B_{\sigma} .
$$

\section{然:}

The idempotent $\eta_{\tau}$ corresponding to $B_{\tau}$ can be gotten by the following construction of Osima [9]: Let the characters of $B_{\tau}$ be $\chi_{\mu}, \mu$ running through an index set $S$. In the element

$$
\frac{1}{g} \sum_{S} \sum_{j=1}^{k} x_{\mu} \chi_{\mu}\left(G_{j}^{-1}\right) K_{j}
$$

of $Z(\xi, \Omega)$, reduce each coefficient modulo $p$; the resulting sum is $\eta_{\tau}$. From (3) it follows that $\alpha$ also permutes the idempotents $\eta_{r}$. Indeed, if (2) holds, then

$$
\alpha: \eta_{\sigma} \rightarrow \eta_{\tau}
$$


Let $\mathfrak{W}$ be a normal subgroup of $\mathbb{S}$, written $\mathfrak{W} \triangleleft \mathfrak{G}$, and let $\mathfrak{A}$ be the group of automorphisms of $\mathfrak{W}$ induced by the elements of $\mathfrak{S}$. We attach a tilde sign $\sim$ to quantities associated with $\mathfrak{W}$. According to the above remarks, $\mathfrak{A}$ has two permutation representations, one permuting the blocks of $\mathfrak{S}$, the other permuting the primitive idempotents of $\mathfrak{B}\left(\mathfrak{S}, \Omega^{*}\right)$. If $\tilde{J}_{1}, \tilde{\mathfrak{J}}_{2}, \cdots, \tilde{\mathfrak{J}}_{n}$ are the systems of transitivity for the blocks in the first representation, replacing $\widetilde{B}_{\tau}$ in each $\tilde{J}_{i}$ by the corresponding $\tilde{\eta}_{\tau}$ gives the systems of transitivity in the second representation. For this reason we use the same letter $\tilde{\mathfrak{J}}_{i}$ in both cases. If $\tilde{B}_{\tau}$ and $\tilde{B}_{\sigma}$ are two blocks of $\mathfrak{S}$ in the same $\tilde{J}_{i}$, then the defect groups of $\widetilde{B}_{\tau}$ and $\widetilde{B}_{\sigma}$ are conjugate in $\mathscr{B} ;$ in particular, $\widetilde{B}_{\tau}$ and $\widetilde{B}_{\sigma}$ have the same defect.

Consider the sum $\epsilon_{i}=\sum \tilde{\eta}_{\tau}$ taken over the idempotents in the set $\tilde{\mathfrak{J}}_{i}$. Since $\tilde{J}_{i}$ is a system of transitivity for the second permutation representation of $\mathfrak{A},\left(\epsilon_{i}\right)^{\alpha}=\epsilon_{i}$ for all $\alpha$ in $\mathfrak{A}$. It follows easily that $\epsilon_{i}$ is a linear combination of the $K_{j}$ over $\Omega^{*}$ and thus belongs to $3\left(\mathcal{B}, \Omega^{*}\right)$. But being a sum of orthogonal idempotents, $\epsilon_{i}$ is itself an idempotent. $\epsilon_{i}$ must then be the sum of certain primitive idempotents of $\mathcal{Z}\left(\mathcal{G}, \Omega^{*}\right)$, say $\epsilon_{i}=\sum \eta_{\sigma}$. Let $\Im_{i}$ be the set of $\eta_{\sigma}$ appearing in $\epsilon_{i}$. We shall also let $J_{i}$ stand for the set of blocks $B_{\sigma}$ of $(\xi)$ which correspond to the $\eta_{\sigma}$ in $J_{i}$. The different $J_{i}$ are disjoint since $\epsilon_{i} \epsilon_{j}=0$ for $i \neq j$, and there is a $1-1$ correspondence

$$
\tilde{J}_{\imath} \leftrightarrow \mathcal{J}_{i} .
$$

Lemma (1B). Let $\mathfrak{S} \triangleleft(\$)$, and let $\chi_{\mu}$ be an irreducible character of $\mathbb{S}$ in the block $B_{\tau}$ in $\mathfrak{J}_{i}$. Then each block of $\mathfrak{S}$ in the corresponding $\tilde{\mathfrak{J}}_{i}$ contains the same number of irreducible characters of $\mathfrak{S}$ which occur as constituents in the restriction $\chi_{\mu} \mid \mathfrak{S}$ of $\chi_{\mu}$ to $\mathfrak{S}$.

Proof. By the results of Clifford in [6], the distinct irreducible constituents of $\chi_{\mu} \mid \mathfrak{S}$ are of the form $\tilde{\chi}_{\mu}^{\alpha}$, where $\tilde{\chi}_{\mu}$ is a fixed irreducible character of $\mathfrak{S}$ and $\alpha$ is in $\mathfrak{A}$. Moreover, every $\tilde{\chi}_{\mu}^{\alpha}$ different from $\tilde{\chi}_{\mu}$ appears as a constituent in $\chi_{\mu} \mid \mathfrak{S}$. It follows that the distinct irreducible constituents of $\chi_{\mu} \mid \mathfrak{S}$ are distributed among the blocks of a certain $\tilde{\mathfrak{J}}_{j}$, each block in $\tilde{\mathfrak{J}}_{j}$ having the same number of constituents. It remains to show $j=i$. Let $\mathfrak{X}_{\mu}^{*}$ be a modular representation gotten from $\mathfrak{X}_{\mu}$ by first writing $\mathfrak{X}_{\mu}$ with coefficients in $\boldsymbol{D}$ and then passing to the residue class field $\Omega^{*}$. Since $\eta_{\tau}$ is a summand in $\epsilon_{i}$,

$$
\mathfrak{X}_{\mu}^{*}\left(\epsilon_{i}\right)=I,
$$

where $I$ is the identity matrix. But $\epsilon_{i}$ is the sum of the idempotents in $\tilde{\mathfrak{J}}_{i}$ as well, and so

$$
\left(\mathfrak{X}_{\mu}^{*} \mid \mathfrak{S}\right)\left(\epsilon_{i}\right)=\sum_{\sigma}\left(\mathfrak{X}_{\mu}^{*} \mid \mathfrak{S}\right)\left(\tilde{\eta}_{\sigma}\right)=I,
$$

where the sum runs over the $\tilde{\eta}_{\sigma}$ in $\tilde{J}_{i}$. This shows that the constituents of $\chi_{\mu} \mid \mathfrak{G}$ belong to the blocks in $\tilde{\mathfrak{J}}_{i}$, and $j=i$. 
LEMMA (1C). Let $\mathfrak{S} \triangleleft(\$)$. Two irreducible characters $\chi_{\lambda}$ and $\chi_{\mu}$ of $\&$ belong to blocks in the same set $J_{i}$ if and only if there exists a chain of irreducible characters of (B)

$$
\chi_{i_{1}}, \chi_{i_{2}}, \cdots, \chi_{i_{2 r-1}}, \chi_{i_{2 r}}
$$

with the following properties: (i) $\chi_{\lambda}=\chi_{i_{1}}$ and $\chi_{\mu}=\chi_{i_{2}}$; (ii) for $j$ odd, $\chi_{i_{j}}$ and $\chi_{i_{j+1}}$ belong to the same block of $\mathfrak{B}$; (iii) for $j$ even, $\chi_{i_{j}} \mid \mathfrak{W}$ and $\chi_{i_{j+1}} \mid \mathfrak{S}$ have a common constituent $\left({ }^{2}\right)$.

Proof. We use the following notation. Let $\zeta$ be a character of $\xi$ : If $\chi_{j}$ is a constituent of $\zeta$, we write $\zeta \supseteq \chi_{j}$. Similarly, if $\phi_{\rho}$ is a modular constituent of $\zeta$, we write $\zeta \supseteq \phi_{\rho}$. This notation will carry over to the characters of $\mathfrak{W}$ as well.

If a chain (5) exists with the properties (i)-(iii), then $\chi_{\lambda}$ and $\chi_{\mu}$ belong to blocks in the same set $\mathfrak{J}_{i}$. For any two adjacent characters in the chain obviously do. Conversely, suppose that $\chi_{\lambda}$ and $\chi_{\mu}$ are in blocks of some $\mathfrak{J}_{i}$. By (1B) an irreducible constituent of $\chi_{\lambda} \mid \mathfrak{S}$ and one of $\chi_{\mu} \mid \mathfrak{S}$ belong to the same block in $\tilde{J}_{i}$. Thus there exist an irreducible modular constituent $\tilde{\phi}_{\rho}$ of $\chi_{\lambda} \mid \mathfrak{S}$ and an irreducible modular constituent $\tilde{\phi}_{\sigma}$ of $\chi_{\mu} \mid \mathfrak{S}$ such that $\tilde{\phi}_{\rho}$ and $\tilde{\phi}_{\sigma}$ are in the same block $\widetilde{B}_{r}$ of $\mathfrak{G}$. We can then find a chain of irreducible modular characters of $\mathfrak{S}$

$$
\tilde{\phi}_{i_{1}}, \tilde{\phi}_{i_{2}}, \cdots, \tilde{\phi}_{i_{\text {s }}}
$$

with the following properties: (i) $\tilde{\phi}_{p}=\tilde{\phi}_{i_{1}}$ and $\tilde{\phi}_{\sigma}=\tilde{\phi}_{i,}$. (ii) There exists an irreducible character $\tilde{\chi}_{i_{j}}$ of $\mathfrak{S}$ such that $\tilde{\chi}_{i_{j}} \supseteq \tilde{\phi}_{i_{j}}$ and $\tilde{\chi}_{i_{j}} \supseteq \tilde{\phi}_{i_{j+1}}, j=1,2, \cdots$, $s-1$. For if this were not so, the matrix of decomposition numbers for the block $\widetilde{B}_{r}$ would decompose in an unpermitted way $[5,(28)]$.

Consider the characters $\zeta_{i_{1}}, \zeta_{i_{2}}, \cdots, \zeta_{i_{8-1}}$ of (S) induced by $\tilde{\chi}_{i_{1}}, \tilde{\chi}_{i_{2}}, \cdots$, $\tilde{\chi}_{i_{s-1}}$. If $\zeta_{i_{j}} \supseteq \chi_{\alpha}$ and $\chi_{\beta}$, then by the Frobenius reciprocity theorem, the two characters $\chi_{\alpha}$ and $\chi_{\beta}$ satisfy condition (iii) of the lemma. Moreover, there exist an irreducible constituent of $\zeta_{i j}$ and an irreducible constituent of $\zeta_{i_{j+1}}$ such that both constituents are in the same block of $\mathcal{B}$. For let $\phi_{i_{j+1}}$ be an irreducible constituent in the modular character of $\mathbb{B}$ induced by $\tilde{\phi}_{i_{j+1}}$. Since $\tilde{\phi}_{i_{j+1}}$ is a modular constituent of both $\tilde{\chi}_{i_{j}}$ and $\tilde{\chi}_{i_{j+1}}$, we have $\zeta_{i_{j}}$ and $\zeta_{i_{j+1}} \supseteq \phi_{i_{j+1}}$. So there exist a $\chi_{i_{j}}$ such that $\zeta_{i_{j}} \supseteq \chi_{i_{j}} \supseteq \phi_{i_{j+1}}$ and a $\chi_{i_{j+1}}$ such that $\zeta_{i_{j+1}} \supseteq \chi_{i_{j+1}}$ $\supseteq \phi_{i_{j+1}}$. In particular, $\chi_{i_{j}}$ and $\chi_{i_{j+1}}$ are in the same block of $B$. This construction can be done successively for $j=1,2, \cdots, s-2$, and after renumbering the characters, we can construct a chain (5) satisfying (ii) and (iii). To see that $\chi_{\lambda}$ and $\chi_{\mu}$ can be fitted in, we need only show that some constituent of $\zeta_{i_{1}}$ is in the same block as $\chi_{\lambda}$, and that some constituent of $\zeta_{i_{d-1}}$ is in the same block as $\chi_{\mu}$. Let $\phi_{\rho}$ and $\phi_{\sigma}$ be modular constituents of $\chi_{\lambda}$ and $\chi_{\mu}$ respectively, such that $\phi_{\rho} \mid \mathfrak{S} \supseteq \tilde{\phi}_{\rho}$ and $\phi_{\sigma} \mid \mathfrak{S} \supseteq \tilde{\phi}_{\sigma}$. Then $\phi_{\rho}$ and $\phi_{\sigma}$ are constituents of the modular characters of $(B)$ induced by $\tilde{\phi}_{\rho}$ and $\tilde{\phi}_{\sigma}$ respectively. Thus some con-

(2) This lemma was suggested by R. Brauer. 
stituent of $\zeta_{i_{1}}$ contains $\phi_{\rho}$ as a modular constituent, and similarly, some constituent of $\zeta_{i_{\varepsilon-1}}$ contains $\phi_{\sigma}$ as a modular constituent.

Lemмa (1D). Let $\mathfrak{S} \triangleleft\left(5\right.$. Suppose a block $B_{\tau}$ of defect $d$ in the set $\mathfrak{J}_{i}$ satisfies the following condition: There is a p-regular element $V$ in $\mathfrak{S}$ such that (i) $V$ has defect $d$ in (B); (ii) $\psi_{\tau}(V) \neq 0$. Then $d$ is the largest defect occurring for the blocks in $\mathfrak{J}_{i}$. Conversely, if $B_{\tau}$ has the largest defect $d$ in $\mathfrak{J}_{i}$, then there exists a p-regular element $V$ in $\mathfrak{S}$ satisfying (i) and (ii).

Proof. We prove the second part first. Let $B_{\rho}$ be a block of largest defect in $\mathfrak{J}_{i}$, say of defect $d_{i}$. Now

$$
\psi_{\rho}\left(\epsilon_{i}\right)=\psi_{\rho}\left(\sum \tilde{\eta}_{\sigma}\right)=1,
$$

where the $\tilde{\eta}_{\sigma}$ are summed over the set $\tilde{J}_{i}$. There is then a $p$-regular element $U$ in $\mathfrak{S}$ appearing in $\epsilon_{i}$ such that $\psi_{\rho}(U) \neq 0$. In particular, $\nu(U) \geqq d_{i}[2,(8 \mathrm{~A})]$. But $\epsilon_{i}$ is also the sum of the idempotents $\eta_{\sigma}$ in $J_{i}$. The construction (3) of $\eta_{\sigma}$ given in [9] shows that the nonzero coefficients in $\eta_{\sigma}$ belong to $p$-regular elements whose defect does not exceed the defect of $B_{\sigma}$. Since $d_{i}$ is the largest defect occurring in $\mathfrak{J}_{i}, \nu(U) \leqq d_{i}$, and this $U$ satisfies (i) and (ii). We shall keep $B_{\rho}$ as this fixed block.

Suppose $B_{\tau}$ is a block in $\Im_{i}$ of defect $d$ such that a $p$-regular element $V$ exists which satisfies (i) and (ii). Let $\chi_{\mu}$ be a character in $B_{\tau}$, and $\chi_{\lambda}$ one in $B_{\rho}$. The restriction of any irreducible character $\chi_{\alpha}$ to $\mathfrak{S}$ decomposes by Clifford's theorem

$$
\chi_{\alpha} \mid \mathfrak{S}=r \sum_{\gamma} \tilde{\chi}_{\gamma}
$$

where the $\tilde{\chi}_{\gamma}$ form a complete set of distinct irreducible characters of $\mathfrak{S}$ conjugate in $\mathfrak{S}^{\text {S }}$, say $m$ in number. Let $\tilde{\omega}_{\gamma}$ be the linear character associated to $\tilde{\chi}_{\gamma}$. Then

$$
\omega_{\alpha}(V)=\frac{((5): \mathfrak{S})}{m} \frac{\tilde{n}(V)}{n(V)} \sum_{\gamma} \tilde{\omega}_{\gamma}(V),
$$

where $\tilde{n}(V)$ is the order of $(\tilde{\mathfrak{R}} V)=\mathfrak{N}(V) \cap \mathfrak{S}$, the normalizer of $V$ in $\mathfrak{S}$. The right hand side of (6) depends only on the class of conjugate characters $\tilde{\chi}_{\gamma}$. Consequently, if $\chi_{\beta}$ is any other irreducible character of $(5)$ such that $\chi_{\beta} \mid \mathfrak{S}$ and $\chi_{\alpha} \mid \mathfrak{S}$ have common constituents, then $\omega_{\beta}(V)=\omega_{\alpha}(V)$. On the other hand, if $\chi_{\beta}$ is any character of $(\xi)$ in the same block as $\chi_{\alpha}$, then $\omega_{\beta}(V) \equiv \omega_{\alpha}(V)(\bmod p)$. Now connect $\chi_{\mu}$ and $\chi_{\lambda}$ by a chain (5) satisfying the properties of (1C). By the above remarks, it follows that

$$
\omega_{\lambda}(V) \equiv \omega_{\mu}(V) \not \equiv 0(\bmod p),
$$

and therefore $d_{i} \leqq \nu(V)=d$. Since we already have $d_{i} \geqq d, d_{i}=d$. 
Corollary (1E). Let $\mathfrak{S} \triangleleft \mathfrak{G}$, and let $B_{\rho}$ be a block of largest defect $d_{i}$ in $\mathfrak{J}_{i}$. Then the defect groups of each $B_{r}$ in $J_{i}$ are contained in the defect groups of $B_{\rho}$. In particular, if $B_{\rho}$ and $B_{\tau}$ have defect $d_{i}$, their defect groups are the same.

Proof. Let $V$ be a $p$-regular element in $\mathfrak{S}$ such that $\nu(V)=d_{i}$ and $\psi_{\rho}(V) \neq 0$. The last part of the proof of $(1 D)$ implies that $\omega_{\mu}(V) \neq 0(\bmod \mathfrak{p})$ for any character $\chi_{\mu}$ in $B_{\tau}$. Thus a defect group of $B_{\tau}$ is contained in a defect group $\mathfrak{D}$ of $V$. But $\mathfrak{D}$ is a defect group of $B_{\rho}$, and this proves the first assertion. The second part follows from the fact that any two defect groups of a block are conjugate.

Theorem (1F). Let $\mathfrak{S} \triangleleft(\$)$, and let $B_{\rho}$ be a block of largest defect $d_{i}$ in $\mathfrak{J}_{i}$. Suppose $\chi_{\mu}$ is a character in $B_{\rho}$ of height 0 , and $\mathfrak{T}$ is the inertial group in $\mathbb{B}$ of an irreducible constituent $\tilde{\chi}_{\alpha}$ of $\chi_{\mu} \mid \mathfrak{S}$, that is,

$$
\mathfrak{T}=\left\{G \text { in } \mathfrak{S}: \tilde{\chi}_{\alpha}\left(G^{-1} X G\right)=\sim_{\alpha}(X) \text { for all } X \text { in } \mathfrak{W}\right\} \text {. }
$$

Then $\mathfrak{T}$ contains a defect group $\mathfrak{D}$ of $B_{\rho}$ and $\mathfrak{D} \mathfrak{W} / \mathfrak{W}$ is a Sylow p-subgroup of $\mathfrak{T} / \mathfrak{S}$. Moreover, $\mathfrak{D} \cap \mathfrak{S}$ is a defect group of some block in the corresponding $\tilde{\mathfrak{J}}_{i}$.

Proof. Let $V$ be a $p$-regular element in $\mathfrak{S}$ such that $\nu(V)=d_{i}$ and $\psi_{\rho}(V) \neq 0$. Then $\omega_{\mu}(V) \not \equiv 0(\bmod p)$ and since $\chi_{\mu}$ has height 0 , it follows that $\chi_{\mu}(V)$ $\not \equiv 0(\bmod \mathfrak{p})$ as well. Let $H_{j}$ be a system of left coset representatives of $\mathfrak{T}$ in $\mathfrak{S}$. We decompose $\mathbb{B}$ into a disjoint union of double cosets modulo $(\mathfrak{D}, \mathfrak{T})$, where $D$ is a fixed defect group of $V$ in $(B)$. Representatives $X_{\beta}$ can be chosen from the $H_{j}$ such that $\left(\mathcal{H}\right.$ is a disjoint union $\bigcup_{\beta} \mathfrak{D} X_{\beta} \mathfrak{T}$. Each double coset $\mathfrak{D} X_{\beta} \mathfrak{T}$ consists of $\left(X_{\beta}^{-1} \mathfrak{D} X_{\beta}: 1\right)(\mathfrak{T}: 1) /\left(X_{\beta}^{-1} \mathfrak{D} X_{\beta} \cap \mathfrak{T}: 1\right)$ elements, and therefore contains $a_{\beta}=\left(X_{\beta}^{-1} \mathfrak{D} X_{\beta}: X_{\beta}^{-1} \mathfrak{D} X_{\beta} \cap \mathfrak{T}\right)$ of the representatives $H_{j}$. If $H_{j}=D X_{\beta} T$, where $D$ is in $\mathfrak{D}$, and $T$ is in $\mathfrak{T}$, then

$$
\begin{aligned}
\tilde{\chi}_{\alpha}\left(H_{j}^{-1} V H_{j}\right) & =\tilde{\chi}_{\alpha}\left(\left(D X_{\beta} T\right)^{-1} V\left(D X_{\beta} T\right)\right) \\
& =\tilde{\chi}_{\alpha}\left(X_{\beta}^{-1} V X_{\beta}\right) .
\end{aligned}
$$

Let $\chi_{\mu} \mid \mathfrak{S}=r \sum_{\gamma} \tilde{\chi}_{\gamma}$ be the decomposition of $\chi_{\mu} \mid \mathfrak{S}$. Then

$$
\chi_{\mu}(V)=r \sum_{\beta} a_{\beta} \tilde{\chi}_{\alpha}\left(X_{\beta}^{-1} V X_{\beta}\right) \not \equiv 0(\bmod \mathfrak{p}) .
$$

In particular, some $a_{\beta} \not \equiv 0(\bmod p)$ and so $X_{\beta}^{-1} \mathfrak{D} X_{\beta} \subseteq \mathfrak{T}$. Therefore $\mathfrak{T}$ contains a defect group of $B_{\rho}$. Replacing $V$ by a suitable conjugate, we may assume that $\mathfrak{D} \subseteq \mathfrak{T}$, where $\mathfrak{D}$ is a defect group of $V$.

The number of distinct conjugates of $\tilde{\chi}_{\alpha}$ is ((S): $\left.\mathfrak{T}\right)$. As a result of the decomposition of $\chi_{\mu} \mid \mathfrak{S}$, we also have

$$
\omega_{\mu}(V)=(\mathfrak{T}: \mathfrak{S}) \frac{\tilde{n}(V)}{n(V)} \sum_{\gamma} \tilde{\omega}_{\gamma}(V) \not \equiv 0(\bmod \mathfrak{p}),
$$

where $\tilde{\omega}_{\gamma}$ is the linear character associated to $\tilde{\chi}_{\gamma}$. Since $\mathfrak{D} \subseteq \mathfrak{T}, \mathfrak{D}$ is contained 
in some Sylow $p$-subgroup $\mathfrak{B}$ of $\mathfrak{T}$. In particular, $\mathfrak{B S} / \mathfrak{Q} \supseteq \mathfrak{D} / \mathfrak{G}$ and $(\mathfrak{B}: \mathfrak{P} \cap \mathfrak{Q}) \geqq(\mathfrak{D}: \mathfrak{D} \cap \mathfrak{Q})$, so that $(\mathfrak{T}: \mathfrak{W}) \tilde{n}(V) / n(V)$ must be a local integer. From (8) it then follows that

$$
(\mathfrak{B}: \mathfrak{B} \cap \mathfrak{T})=(\mathfrak{D}: \mathfrak{D} \cap \mathfrak{S}), \quad \tilde{\omega}_{\gamma}(V) \not \equiv 0(\bmod \mathfrak{p}) \text { for some } \gamma .
$$

The first equation shows that $\mathfrak{D} \mathfrak{Q} / \mathfrak{W}$ is a Sylow $p$-subgroup of $\mathfrak{T} / \mathfrak{W}$. The second shows that $\mathfrak{D} \cap \mathfrak{E}$ contains a defect group of a block in $\tilde{\mathfrak{J}}_{i}$, and consequently, $\nu(\tilde{n}(V)) \geqq \bar{d}$, where $\bar{d}$ is the common defect of the blocks in $\tilde{\mathfrak{J}}_{i}$. But from (7), some $\tilde{\chi}_{\alpha}\left(X_{\beta}^{-1} V X_{\beta}\right) \not \equiv 0(\bmod \mathfrak{p})$ and so $\tilde{d} \geqq \nu(\tilde{n}(V))$ by (1). Thus $\mathfrak{D} \cap \mathfrak{S}$ is a defect group of a block in $\tilde{J}_{i}$.

2. The reduction step for the case $p \nmid(\mathfrak{g}: 1)$. Let $\mathfrak{G}$ be a normal $p^{\prime}$-subgroup of $\$$. Under this condition we shall correlate the modular invariants of any given block $B_{\tau}$ of $B$ to those of a suitable block of another group of somewhat simpler structure. In the case where $\&$ is $p$-solvable, this new group will also be $p$-solvable.

The modular theory for the $p^{\prime}$-group $\mathfrak{S}$ collapses. As a result, the irreducible characters of $\mathfrak{S}$ are also the irreducible modular characters of $\mathfrak{E}$, and each irreducible character forms a block by itself. For simplicity, we change our notation and denote the irreducible characters of $\mathfrak{E}$ by $\left\{\theta_{\rho}\right\}$. Each block of $\mathfrak{E}$ will be identified with the unique $\theta_{\rho}$ contained in it. We retain, however, the other notational conventions of $\S 1$. A set $\tilde{\jmath}_{i}$ is then a class $\left\{\theta_{\rho}\right\}$ of irreducible characters of $\mathfrak{S}$ associated in $(\mathfrak{S})$ to a given $\theta_{p}$. It follows easily that if $\mathfrak{J}_{i} \leftrightarrow \tilde{\mathfrak{J}}_{i}$ $=\left\{\theta_{\rho}\right\}$, then a character $\chi_{\mu}$ of $\leftrightarrow$ is in a block of $\Im_{i}$ if and only if

$$
\chi_{\mu} \mid \mathfrak{G}=r_{\mu} \sum \theta_{\sigma},
$$

where the $\theta_{\sigma}$ are in $\left\{\theta_{\rho}\right\}$.

Consider a fixed character $\theta_{\rho}$, the set $\mathfrak{J}_{i}$ corresponding to $\tilde{\mathfrak{J}}_{i}=\left\{\theta_{\rho}\right\}$, and a block $B_{r}$ in $\exists_{i}$. Let $\mathfrak{T}$ be the inertial group of $\theta_{\rho}$ in $\mathfrak{B}$, and let

$$
h=(\mathfrak{S}: 1), \quad t=(\mathfrak{T}: 1) .
$$

By (1E) and (1F), a defect group of $B_{\tau}$ is contained in $\mathfrak{T}$. Because of (9) each character $\chi_{\mu}$ in $B_{r}$ determines a unique irreducible character $\chi_{\mu}^{\prime}$ of $\mathfrak{T}$ with the following properties: (i) $\chi_{\mu}^{\prime} \mid \mathfrak{S}=r_{\mu} \theta_{\rho}$; (ii) the character of $\mathbb{B}$ induced by $\chi_{\mu}^{\prime}$ is $\chi_{\mu}$. These characters $\chi_{\mu}^{\prime}$ arise by considering certain systems of imprimitivity for $\mathfrak{X}_{\mu}$; see Clifford [6].

LEMma (2A). The set of all characters $\chi_{\mu}^{\prime}$ of $\mathfrak{T}$ arising from all the characters $\chi_{\mu}$ in $B_{\tau}$ is a block $B_{\tau}^{\prime}$ of $\mathfrak{T}$. If $d$ is the defect of $B_{r}$, then $d$ is also the defect of $B_{\tau}^{\prime}$ and a suitable defect group of $B_{\tau}$ is one for $B_{\tau}^{\prime}$.

Proof. Let $\chi_{\lambda}$ be a fixed character of height 0 in $B_{r}$. Then $x_{\lambda}=r_{\lambda}(\mathfrak{S}: \mathfrak{T}) \theta_{\rho}(1)$, where $r_{\lambda}$ is the integer appearing in (9). Since $\theta_{\rho}(1)$ is not divisible by $p$, we have 


$$
\begin{aligned}
a-d & =\nu\left(r_{\lambda}\right)+\nu(\mathbb{S}: \mathfrak{T}) \\
& =\nu\left(r_{\lambda}\right)+a-\delta,
\end{aligned}
$$

where $\delta=\nu(\mathfrak{T}: 1)=\nu(t)$. Therefore $\nu\left(r_{\lambda}\right)=\delta-d$, and from degree $\chi_{\lambda}^{\prime}=x_{\lambda}^{\prime}$ $=r_{\lambda} \theta_{\rho}(1)$, we see that $\chi_{\lambda}^{\prime}$ belongs to a block $B^{\prime}$ of $\mathfrak{T}$ of defect $\geqq d$.

Let $\left\{G_{\alpha}\right\}$ be a set of left coset representatives of $\mathfrak{T}$ in $\mathcal{G}$. Since $\chi_{\lambda}^{\prime}$ induces $\chi_{\lambda}$, it follows that for $V$ in $(3)$,

$$
\chi_{\lambda}(V)=\sum_{\alpha} \chi_{\lambda}^{\prime}\left(G_{\alpha}^{-1} V G_{\alpha}\right)
$$

where the sum is extended only over those $\alpha$ such that $G_{\alpha}^{-1} V G_{\alpha}$ is in $\mathfrak{T}$. Suppose $V$ is a $p$-regular element of $\xi$ such that $\omega_{\lambda}(V) \neq 0(\bmod \mathfrak{p})$, and such that a defect group $\mathfrak{D}$ of $V$ in $\mathfrak{B}$ is a defect group of $B_{\tau}$. Then

$$
\omega_{\lambda}(V)=\sum_{\alpha} \omega_{\lambda}^{\prime}\left(G_{\alpha}^{-1} V G_{\alpha}\right) \frac{n^{\prime}\left(G_{\alpha}^{-1} V G_{\alpha}\right)}{n(V)},
$$

where $\omega_{\lambda}^{\prime}$ is the class character of $\chi_{\lambda}^{\prime}$, and $n^{\prime}\left(G_{\alpha}^{-1} V G_{\alpha}\right)=\left(\mathfrak{T} \cap \mathfrak{N}\left(G_{\alpha}^{-1} V G_{\alpha}\right): 1\right)$ is the order of the normalizer of $G_{\alpha}^{-1} V G_{\alpha}$ in $\mathfrak{T}$. Consider the double cosets $(\mathfrak{I}, \mathfrak{I})$. Representatives $H_{\alpha}$ of these can be chosen from the $G_{\beta}$. If $H_{\alpha}^{-1} V H_{\alpha}$ is in $\mathfrak{T}$ and $G_{\beta}=D H_{\alpha} T$ with $T$ in $\mathfrak{T}$ and $D$ in $\mathfrak{D}$, then $G_{\beta}^{-1} V G_{\beta}$ is in $\mathfrak{T}$ as well. Thus the sum in (10) can be arranged over the representatives $G_{\beta}$ in certain double cosets of $(\mathfrak{D}, \mathfrak{T})$. Suppose the coset of $H_{\alpha}$ contains $a_{\alpha}$ representatives $G_{\beta}$. For such a $G_{\beta}$, we have $\omega_{\lambda}^{\prime}\left(G_{\beta}^{-1} V G_{\beta}\right)=\omega_{\lambda}^{\prime}\left(H_{\alpha}^{-1} V H_{\alpha}\right)$. The sum (10) can then be rewritten as

$$
\omega_{\lambda}(V)=\sum_{\alpha} b_{\alpha} \omega_{\lambda}^{\prime}\left(H_{\alpha}^{-1} V H_{\alpha}\right)
$$

where $b_{\alpha}=a_{\alpha}\left(n^{\prime}\left(H_{\alpha}^{-1} V H_{\alpha}\right) / n(V)\right)$. Since $a_{\alpha}=\left(H_{\alpha}^{-1} \mathfrak{D} H_{\alpha}: H_{\alpha}^{-1} \mathfrak{D} H_{\alpha} \cap \mathfrak{T}\right)$ and $\nu\left(n^{\prime}\left(H_{\alpha}^{-1} V H_{\alpha}\right)\right) \geqq \nu\left(H_{\alpha}^{-1} \mathfrak{D} H_{\alpha} \cap \mathfrak{T}: 1\right)$, the $b_{\alpha}$ are actually local integers. We can then assert that there is some $\alpha$ such that

$$
b_{\alpha} \not \equiv 0(\bmod p), \quad \omega_{\lambda}^{\prime}\left(H_{\alpha}^{-1} V H_{\alpha}\right) \not \equiv 0(\bmod \mathfrak{p}) .
$$

In particular, $\nu\left(n^{\prime}\left(H_{\alpha}^{-1} V H_{\alpha}\right)\right)=\nu\left(H_{\alpha}^{-1} \mathfrak{D} H_{\alpha} \cap \mathfrak{T}: 1\right) \leqq d$. This shows that $B^{\prime}$, the block of $\mathfrak{T}$ containing $\chi_{\lambda}^{\prime}$, has defect $\leqq d$. Since its defect was shown earlier to be $\geqq d, B^{\prime}$ has defect $d$. Therefore $\nu\left(H_{\alpha}^{-1} \mathfrak{D} H_{\alpha} \cap \mathfrak{T}: 1\right)=d$ and $H_{\alpha}^{-1} \mathfrak{D} H_{\alpha} \subseteq \mathfrak{T}$. Replacing $\mathfrak{D}$ by the conjugate $H_{\alpha}^{-1} \mathfrak{D} H_{\alpha}$, we may assume that $\mathfrak{D}$ is a defect group of $B^{\prime}$.

We now apply the remarks at the beginning of this section to the situation $\mathfrak{S} \triangleleft \mathfrak{T}$. Let $\chi_{\mu}^{\prime}$ be any character of $\mathfrak{T}$ in the block $B^{\prime}$. The restriction $\chi_{\mu}^{\prime} \mid \mathfrak{S}$ must be of the form $r_{\mu} \theta_{\rho}$, and so $\chi_{\mu}^{\prime}$ induces an irreducible character $\chi_{\mu}$ of $\$ S$. Consider the sum (10) with $\lambda$ replaced by $\mu$. Since the derivation of (11) from (10) depended only on the quantities $V, \mathfrak{D}, \mathfrak{T}$, and $\theta_{\rho},(11)$ is valid when $\lambda$ is replaced by $\mu$. Now $\omega_{\mu}^{\prime}(V) \equiv \omega_{\lambda}^{\prime}(V)(\bmod \mathfrak{p})$; it then follows that $\omega_{\mu}(V)$ 
$\equiv \omega_{\lambda}(V)(\bmod \mathfrak{p})$ for all $p$-regular elements $V$ with $\mathfrak{D}$ as defect group. In particular, $\chi_{\mu}$ belongs to a block $B_{\sigma}$ of $\&$ of defect $\leqq d$ and defect group $\mathfrak{D}_{\sigma} \subseteq \mathfrak{D}$. Let $U$ be a $p$-regular element in $\&$ with $\mathfrak{D}_{\sigma}$ as defect group such that $\omega_{\mu}(U) \not \equiv 0(\bmod \mathfrak{p})$. If we use the double cosets of $\left(\mathfrak{D}_{\sigma}, \mathfrak{T}\right)$ and proceed as before, we can derive that

$$
\begin{aligned}
& \omega_{\lambda}(U)=\sum_{\alpha} c_{\alpha} \omega_{\lambda}^{\prime}\left(H_{\alpha}^{-1} U H_{\alpha}\right), \\
& \omega_{\mu}(U)=\sum_{\alpha} c_{\alpha} \omega_{\mu}^{\prime}\left(H_{\alpha}^{-1} U H_{\alpha}\right),
\end{aligned}
$$

where the $c_{\alpha}$ are local integers and the $H_{\alpha}$ are certain representatives of the double cosets $\left(D_{\sigma}, \mathfrak{T}\right)$. From this we see that $\omega_{\lambda}(U) \equiv \omega_{\mu}(U) \not \equiv 0(\bmod \mathfrak{p})$ and therefore $B_{\tau}$ and $B_{\sigma}$ have $\mathfrak{D}$ as defect group. Since $\omega_{\mu}(V) \equiv \omega_{\lambda}(V)(\bmod \mathfrak{p})$ for all $p$-regular elements $V$ with $\mathfrak{D}$ as defect group, it follows from [2, (6F)] that $B_{\sigma}=B_{r}$.

Let $B$ be the set of all the irreducible characters $\chi_{\mu}$ of $\&$ induced by the $\chi_{\mu}^{\prime}$ in $B^{\prime}$. We have just shown that $B \subseteq B_{r}$. If we can show $B$ is a block of $B$, then $B=B_{r}$, and the set $B_{r}^{\prime}$ of the statement is simply $B^{\prime}$. Let $P$ be a $p$ singular element of $B$, $R$ a $p$-regular element of $B$. Then

$$
\begin{aligned}
\sum_{B} \chi_{\mu}(R) \chi_{\mu}(P) & =\sum_{B^{\prime}}\left(\sum_{\alpha} \chi_{\mu}^{\prime}\left(G_{\alpha}^{-1} R G_{\alpha}\right)\right)\left(\sum_{\beta} \chi_{\mu}^{\prime}\left(G_{\beta}^{-1} P G_{\beta}\right)\right) \\
& =\sum_{\alpha, \beta}\left(\sum_{B^{\prime}} \chi_{\mu}^{\prime}\left(G_{\alpha}^{-1} R G_{\alpha}\right) \chi_{\mu}^{\prime}\left(G_{\beta}^{-1} P G_{\beta}\right)\right),
\end{aligned}
$$

where the sum is only over those $\alpha$ such that $G_{\alpha}^{-1} R G_{\alpha}$ is in $\mathfrak{I}$ and those $\beta$ such that $G_{\beta}^{-1} P G_{\beta}$ is in $\mathfrak{T}$. Each of the sums $\sum_{B^{\prime}} \chi_{\mu}^{\prime}\left(G_{\alpha}^{-1} R G_{\alpha}\right) \chi_{\mu}^{\prime}\left(G_{\beta}^{-1} P G_{\beta}\right)=0$ by the block orthogonality relations $[3,(7 \mathrm{C})]$. Thus

$$
\sum_{B} \chi_{\mu}(R) \chi_{\mu}(P)=0
$$

and by a theorem of Osima [9, Theorem 3], this implies that $B$ is a union of blocks of $B$, and is therefore $B_{r}$. This completes the proof.

We note that the 1-1 correspondence $\chi_{\mu} \leftrightarrow \chi_{\mu}^{\prime}$ is height-preserving, for the height of both $\chi_{\mu}$ and $\chi_{\mu}^{\prime}$ is $\nu\left(r_{\mu}\right)-(\delta-d)$. Let $\phi_{\alpha}$ be an irreducible modular character of $\mathfrak{G} . \phi_{\alpha}$ is in a block of $J_{i}$ if and only if $\phi_{\alpha} \mid \mathfrak{S}$ has the form

$$
\phi_{\alpha} \mid \mathfrak{S}=s_{\alpha} \sum \theta_{\sigma}, \quad \theta_{\sigma} \text { in }\left\{\theta_{\rho}\right\} .
$$

As Clifford's results are still valid for this case, we see that each $\phi_{\alpha}$ in $B_{r}$ determines a unique irreducible modular character $\phi_{\alpha}^{\prime}$ of $\mathfrak{T}$ such that (i) $\phi_{\alpha}^{\prime} \mid \mathfrak{E}$ $=s_{\alpha} \theta_{\rho}$; (ii) the modular character of \& induced by $\phi_{\alpha}^{\prime}$ is $\phi_{\alpha}$. Suppose

$$
\chi_{\mu}^{\prime}=\sum_{\alpha} d_{\mu \alpha}^{\prime} \phi_{\alpha}^{\prime}
$$


is the modular decomposition of a character $\chi_{\mu}^{\prime}$ in $B_{\tau}^{\prime}$. Since $\chi_{\mu}^{\prime} \mid \mathfrak{S}=r_{\mu} \theta_{\rho}$, the $\phi_{\alpha}^{\prime}$ appearing in (12) necessarily satisfy (i) and will induce an irreducible modular character $\phi_{\alpha}$ of $\left(\xi\right.$. Consider the values of the character $\chi_{\mu}$ of $(S)$ induced by $\chi_{\mu}^{\prime}$ for $p$-regular elements. This can be computed in two ways, either by taking $\sum d_{\mu \alpha}^{\prime} \phi_{\alpha}^{\prime}$ and inducing up to $\left(\mathcal{S}\right.$, or by decomposing $\chi_{\mu}$ into its modular constituents. For $p$-regular elements we have the identity

$$
\chi_{\mu}=\sum_{\alpha} d_{\mu \alpha} \phi_{\alpha}=\sum_{\alpha} d_{\mu \alpha}^{\prime} \phi_{\alpha}
$$

Since the $\phi_{\alpha}$ are linearly independent, $d_{\mu \alpha}=d_{\mu \alpha}^{\prime}$. In particular, the correspondence $\phi_{\alpha} \leftrightarrow \phi_{\alpha}^{\prime}$ is 1-1 between the modular characters of $B_{\tau}$ and $B_{\tau}^{\prime}$. If $c_{\alpha \beta}$ and $c_{\alpha \beta}^{\prime}$ are the Cartan invariants relating $\phi_{\alpha}$ and $\phi_{\beta}, \phi_{\alpha}^{\prime}$ and $\phi_{\beta}^{\prime}$ respectively, then $c_{\alpha \beta}^{\prime}=\sum d_{\mu \alpha}^{\prime} d_{\mu \beta}^{\prime}=\sum d_{\mu \alpha} d_{\mu \beta}=c_{\alpha \beta}$. Combining these results together, we have

THEOREM (2B). Let (S) be a group with a nontrivial normal $p^{\prime}$-subgroup $\mathfrak{W}$. Let $B_{\tau}$ be a block of $\left(\mathfrak{S}\right.$. Then there is a subgroup $\mathfrak{T}, \mathfrak{H} \supseteq \mathfrak{T} \supseteq \$$, and a block $B_{\tau}^{\prime}$ of $\mathfrak{T}$, such that the following are true: (i) $B_{\tau}$ and $B_{r}^{\prime}$ have a defect group in common. (ii) There is a 1-1 height-preserving correspondence $\chi_{\mu} \leftrightarrow \chi_{\mu}^{\prime}$ between the ordinary characters $\chi_{\mu}$ in $B_{\tau}$ and the ordinary characters $\chi_{\mu}^{\prime}$ in $B_{\tau}^{\prime}$. (iii) There is a 1-1 correspondence $\phi_{\alpha} \leftrightarrow \phi_{\alpha}^{\prime}$ between the modular characters $\phi_{\alpha}$ in $B_{\tau}$ and the modular characters $\phi_{\alpha}^{\prime}$ in $B_{\tau}^{\prime}$. (iv) With respect to these correspondences of characters, the matrices of decomposition numbers and Cartan invariants of $B_{\tau}$ and $B_{\tau}^{\prime}$ are the same.

The reduction contained in (2B) cannot be used successfully by itself, since it may happen that $\mathfrak{T}=\mathfrak{G}$. Thus the situation $\mathfrak{S} \triangleleft \mathfrak{T}$ must be examined in more detail. Continuing with the notation of this section, let $B_{\tau}^{\prime}$ be the block of $\mathfrak{T}$ obtained from $B_{\tau}$. Each character $\chi_{\mu}^{\prime}$ in $B_{\tau}^{\prime}$, when restricted to $\mathfrak{S}$, has the form $\chi_{\mu}^{\prime} \mid \mathfrak{S}=r_{\mu} \theta_{\rho}$, where $\theta_{\rho}$ depends only on $B_{r}^{\prime}$. If $\mathfrak{X}_{\mu}^{\prime}$ is a representation of $\mathfrak{T}$ whose character is $\chi_{\mu}^{\prime}$, then by Theorem 3 of [6] $\mathfrak{X}_{\mu}^{\prime}$ is the product of two projective representations $\mathfrak{A}_{\mu} \times \mathfrak{B}$ of $\mathfrak{T}$. The following facts are true: $\mathfrak{B}$ is an irreducible $\epsilon^{-1}$ projective representation of $\mathfrak{T}$ with the factor set $\epsilon^{-1}$ and degree $\theta_{\rho}(1) . \mathfrak{A}_{\mu}$ is an irreducible $\epsilon$ projective representation of $\mathfrak{T} / \mathfrak{S}$ with factor set $\epsilon$ and degree $r_{\mu}$. We may first assume that $\mathfrak{B}$ is determined by the block $B_{\tau}^{\prime}$ up to similarity transformations and change of factor set by cohomologous ones. If $\mathfrak{A}_{\mu}$ is any irreducible $\epsilon$ projective representation of $\mathfrak{T} / \mathfrak{S}$, then the product $\mathfrak{A}_{\mu} \times \mathfrak{P}$ is an irreducible representation of $\mathfrak{T}$, though not necessarily in the block $B_{r}^{\prime}$. The factor set $\epsilon$ can be viewed in two ways, either as a factor set of $\mathbb{S} / \mathfrak{W}$, or by inflation as a factor set of $\mathbb{G}$. Let $s$ be the order of its cohomology class in $H^{2}(\mathbb{S} / \mathfrak{S})$ and $s^{\prime}$ the order of its cohomology class

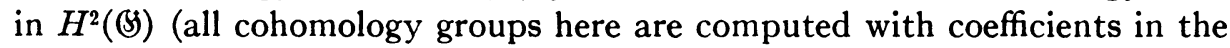
multiplicative subgroup of $\Omega$ and trivial action). From the exact inflationrestriction sequence 


$$
\rightarrow \mathrm{H}^{1}(\mathfrak{S})^{\leftrightarrow} \rightarrow H^{2}(\mathfrak{S} / \mathfrak{S}) \rightarrow \mathrm{H}^{2}(\mathfrak{S})
$$

it follows that $s \mid s^{\prime} h$ since the order of $H^{1}(\mathfrak{W})^{\mathscr{G}}$ is a divisor of $h$. However, $s^{\prime}$ divides $\operatorname{deg} \mathfrak{B}=\operatorname{deg} \theta_{\rho}$ by $[11,(2)]$ and $\operatorname{deg} \theta_{\rho} \operatorname{divides} h$. Therefore $s \mid h^{2}$ and in particular, $(p, s)=1$. Enlarging the field $\Omega$ by a finite extension and using equivalent projective representations if necessary, we may assume that $\epsilon$ consists of sth roots of unity. We will construct a group $\mathfrak{M}$ of order $m=t s / h$ with the following properties: (i) There is a cyclic group $\&$ of order $s$ in the center of $\mathfrak{M}$ such that $\mathfrak{M} / \mathfrak{E} \simeq \mathfrak{T} / \mathfrak{S}$. (ii) The irreducible projective representations $\mathfrak{A}_{\mu}$ of $\mathfrak{T} / \mathfrak{W}$ can be induced by ordinary irreducible representations of $\mathfrak{M}$. Indeed, let $\mathfrak{F}$ be the character group of the cyclic group generated by the factor set $\epsilon$. For any two elements $G, H$ in $\mathfrak{T} / \mathfrak{S}$, define $M_{G, H}$ in $\mathfrak{E}$ to be the character defined by the mapping $\epsilon^{i} \rightarrow \epsilon_{G, H}^{i}$. This gives a factor set of $\mathfrak{T} / \mathfrak{S}$ in $\mathfrak{F}$. Let $\mathfrak{M}$ be the extension of $\mathbb{F}$ by $\mathfrak{T} / \mathfrak{S}$ with this factor set and trivial action on $\mathfrak{F}$. This $\mathfrak{M}$ then satisfies (i) and (ii). To check (ii), we let $\mathfrak{M}$ be represented by the symbols $(E, G)$, where $E$ is in $\mathscr{E}, G$ is in $\mathfrak{T} / \mathfrak{F}$, under the usual rules for multiplication. If $\mathfrak{A}_{\mu}$ is an irreducible $\epsilon$ projective representation of $\mathfrak{T} / \mathfrak{S}$, the representation $\mathfrak{X}_{\mu}^{\prime \prime}$ of $\mathfrak{M}$ defined by setting

$$
\mathfrak{X}_{\mu}^{\prime \prime}(E, G)=\epsilon(E) \mathfrak{A}_{\mu}(G),
$$

will induce $\mathfrak{A}_{\mu}$. Conversely, an irreducible representation $\mathfrak{X}_{\mu}^{\prime \prime}$ of $\mathfrak{M}$ will induce an irreducible $\epsilon$ projective representation of $\mathfrak{T} / \mathfrak{S}$ if the linear character induced by $\mathfrak{X}_{\mu}^{\prime \prime}$ on $\mathcal{E}$ can be identified with $\epsilon$ under the canonical isomorphism between a group and the character group of its character group. For each representation $\mathfrak{A}_{\mu}$ arising from a representation $\mathfrak{X}_{\mu}^{\prime}$ in $B_{\tau}^{\prime}$, let $\mathfrak{X}_{\mu}^{\prime \prime}$ be an irreducible representation of $\mathfrak{M}$ which induces $\mathfrak{A}_{\mu}$. $\mathfrak{X}_{\mu}^{\prime \prime}$ is then determined up to equivalence. The characters of $\mathfrak{A}_{\mu}, \mathfrak{X}_{\mu}^{\prime \prime}$, and $\mathfrak{B}$ are well-defined and will be denoted by $\alpha_{\mu}, \chi_{\mu}^{\prime \prime}$, and $\beta$ respectively. By referring to the characters of $\mathfrak{M}$, we see that the $\alpha_{\mu}$ are always local integers. In a similar way, we see that $\beta$ is always a local integer.

Lemma (2C). The set of all characters $\chi_{\mu}^{\prime \prime}$ of $\mathfrak{M}$ arising from all the characters $\chi_{\mu}^{\prime}$ in $B_{\tau}^{\prime}$ is a block $B_{\tau}^{\prime \prime}$ of $\mathfrak{M}$. If $d$ is the defect of $B_{\tau}^{\prime}$, then $d$ is also the defect of $B_{\tau}^{\prime \prime}$, and the defect groups of $B_{\tau}^{\prime}$ and $B_{\tau}^{\prime \prime}$ are isomorphic.

Proof. We use the following notation: Let $\overline{\mathfrak{T}}=\mathfrak{T} / \mathfrak{S}, \pi$ be the natural mapping $\pi: \mathfrak{M} \rightarrow \overline{\mathfrak{T}}, f$ the natural mapping $f: \mathfrak{T} \rightarrow \overline{\mathfrak{T}}$. $\overline{\mathfrak{S}}, \overline{\mathfrak{F}}, \cdots$ will be elements of $\overline{\mathfrak{T}}$. For each $\bar{G}$ in $\overline{\mathfrak{T}}$, let $\rho(\bar{G})$ be the representative $(1, \bar{G})$ in $\mathfrak{M}$. For the elements $\rho(\bar{G})$ of $\mathfrak{M}$, we have $\chi_{\mu}^{\prime \prime}(\rho(\bar{G}))=\alpha_{\mu}(\bar{G})$. If $\bar{G}$ is $p$-regular, $\rho(\bar{G})$ will also be $p$-regular. To see this, let $c$ be the order of $\bar{G}$; then $(\rho(\bar{G}))^{c}$ belongs to $₹$. Since $c$ and (\&:1) are both prime to $p, \rho(\bar{G})$ must have order prime to $p$. The normalizers in the groups $\mathfrak{M}, \mathfrak{T}, \overline{\mathfrak{T}}$ will be denoted by $\mathfrak{N}^{\prime \prime}, \mathfrak{N}^{\prime}, \overline{\mathfrak{N}}$ respectively, their orders by $n^{\prime \prime}, n^{\prime}, \bar{n}$ respectively. 
Let $\bar{V}$ be any element in $\overline{\mathfrak{T}}$, and set $V^{\prime \prime}=\rho(\bar{V})$. We first show that $\nu(\bar{n}(\bar{V}))$ $=\nu\left(n^{\prime \prime}\left(V^{\prime \prime}\right)\right)$. Let $\mathfrak{R}$ be the subgroup of $\mathfrak{M}$ defined by $\mathfrak{R}=\pi^{-1}(\overline{\mathfrak{R}}(\bar{V}))$. Since $\mathfrak{L} / \mathfrak{F} \simeq \overline{\mathfrak{N}}(\bar{V})$ and $p \nmid(\mathbb{E}: 1)$, it follows that $\nu(\mathbb{R}: 1)=\nu(\bar{n}(\bar{V}))$. We know $\nu(\mathfrak{R}: 1)$ $\geqq \nu\left(n^{\prime \prime}\left(V^{\prime \prime}\right)\right)$ from the inclusion $\mathfrak{N}^{\prime \prime}\left(V^{\prime \prime}\right) \subseteq \mathfrak{R}$. Let $G^{\prime \prime}$ be any element in $\mathfrak{R}$ of order a $p$-power. By definition $G^{\prime \prime} V^{\prime \prime}=V^{\prime \prime} G^{\prime \prime} E^{\prime \prime}$, where $E^{\prime \prime}$ is an element in छ. We rewrite this as $\left(V^{\prime \prime}\right)^{-1} G^{\prime \prime} V^{\prime \prime}=G^{\prime \prime} E^{\prime \prime}$. The element on the left hand side has order a power of $p$; the element on the right hand side has order equal to (order of $G^{\prime \prime}$ ) $\times$ (order of $E^{\prime \prime}$ ). Since $E^{\prime \prime}$ has order prime to $p$, this implies that $E^{\prime \prime}=1$, and $G^{\prime \prime}$ is in $\mathfrak{R}^{\prime \prime}\left(V^{\prime \prime}\right)$. Therefore the Sylow $p$-subgroups of $\mathfrak{Q}$ are contained in $\mathfrak{N}^{\prime \prime}\left(V^{\prime \prime}\right)$ and indeed $\nu(\bar{n}(\bar{V}))=\nu\left(n^{\prime \prime}\left(V^{\prime \prime}\right)\right)$. In particular, the defect groups of $\bar{V}$ in $\overline{\mathfrak{T}}$ and the defect groups of $V^{\prime \prime}$ in $\mathfrak{M}$ are isomorphic.

We proceed with the proof proper. Let $\chi_{\lambda}^{\prime}$ be a character in $B_{\tau}^{\prime}$ of height 0 . The degree $x_{\lambda}^{\prime \prime}$ of $\chi_{\lambda}^{\prime \prime}$ is equal to $\alpha_{\lambda}(1)$. Since $\nu\left(x_{\lambda}^{\prime}\right)=\nu\left(\alpha_{\lambda}(1)\right)=\delta-d$ and $\nu(m)=\delta$, the character $\chi_{\lambda}^{\prime \prime}$ must belong to a block $B^{\prime \prime}$ of $\mathfrak{M}$ of defect $\geqq d$. If $\mathfrak{D}$ is the defect group of $B_{\tau}^{\prime}$, there exists a $p$-regular element $V^{\prime}$ in $\mathfrak{T}$ with $\mathfrak{D}$ as defect group such that $\omega_{\lambda}^{\prime}\left(V^{\prime}\right) \not \equiv 0(\bmod \mathfrak{p})$. Consequently $\chi_{\lambda}^{\prime}\left(V^{\prime}\right)$ $=\alpha_{\lambda}\left(V^{\prime}\right) \beta\left(V^{\prime}\right) \not \equiv 0(\bmod \mathfrak{p})$, and in particular, $\alpha_{\lambda}\left(V^{\prime}\right) \not \equiv 0(\bmod \mathfrak{p})$. Consider

$$
\omega_{\lambda}^{\prime}\left(V^{\prime}\right)=\frac{t}{n^{\prime}\left(V^{\prime}\right)} \frac{\alpha_{\lambda}\left(V^{\prime}\right) \beta\left(V^{\prime}\right)}{\alpha_{\lambda}(1) \beta(1)} \not \equiv 0(\bmod p) .
$$

$\beta\left(V^{\prime}\right) / \beta(1)$ is a local integer since $\beta$ has degree prime to $p$.

$$
\frac{t}{n^{\prime}\left(V^{\prime}\right)} \frac{\alpha_{\lambda}\left(V^{\prime}\right)}{\alpha_{\lambda}(1)}
$$

is a local integer as well, since $\nu(t)-\nu\left(n^{\prime}\left(V^{\prime}\right)\right)+\nu\left(\alpha_{\lambda}\left(V^{\prime}\right)\right)-\nu\left(\alpha_{\lambda}(1)\right)=\delta-d$ $-(\delta-d)=0$. It follows from (13) that

$$
\frac{t}{n^{\prime}\left(V^{\prime}\right)} \frac{\alpha_{\lambda}\left(V^{\prime}\right)}{\alpha_{\lambda}(1)} \neq \equiv 0(\bmod \mathfrak{p}), \quad \frac{\beta\left(V^{\prime}\right)}{\beta(1)} \not \equiv 0(\bmod \mathfrak{p})
$$

As $\mathfrak{A}_{\lambda}$ is actually a projective representation of $\overline{\mathfrak{T}}$, the first part of (14) may be written

$$
\frac{t}{n^{\prime}\left(V^{\prime}\right)} \frac{\alpha_{\lambda}(\bar{V})}{\alpha_{\lambda}(1)} \not \equiv 0(\bmod \mathfrak{p}),
$$

where $\bar{V}=f\left(V^{\prime}\right)$. We must compare the $p$-components of $n^{\prime}\left(V^{\prime}\right)$ and $\bar{n}(\bar{V})$. We know already that $\nu(\bar{n}(\bar{V})) \geqq \nu\left(n^{\prime}\left(V^{\prime}\right)\right)=d$ from the inclusion $\mathfrak{N}^{\prime}\left(V^{\prime}\right) \mathfrak{E} / \mathfrak{E}$ $\subseteq \overline{\mathfrak{N}}(\bar{V})$. But in fact equality holds. For rewrite (15) as

$$
\left[\frac{m}{n^{\prime \prime}\left(V^{\prime \prime}\right)} \frac{\alpha_{\lambda}(\bar{V})}{\alpha_{\lambda}(1)}\right] \frac{n^{\prime \prime}\left(V^{\prime \prime}\right)}{n^{\prime}\left(V^{\prime}\right)} \frac{h}{s} \not \equiv 0(\bmod \mathfrak{p}),
$$

where $V^{\prime \prime}=\rho(\bar{V})$. The term in the brackets can differ only by a multiple of 
an $s$ th root of unity from $\omega_{\lambda}^{\prime \prime}\left(V^{\prime \prime}\right)$, where $\omega_{\lambda}^{\prime \prime}$ is the linear character associated to $\chi_{\lambda}^{\prime \prime}$. (Because of the choice of the representative $\rho(\bar{V})$, this root of unity is actually one.) From the fact $\omega_{\lambda}^{\prime \prime}\left(V^{\prime \prime}\right)$ is a local integer and $\nu\left(\alpha_{\lambda}(\bar{V})\right)=0$, we must have in particular

$$
\nu(m)-\nu\left(n^{\prime \prime}\left(V^{\prime \prime}\right)\right)-\nu\left(\alpha_{\lambda}(1)\right) \geqq 0,
$$

or $\nu\left(n^{\prime \prime}\left(V^{\prime \prime}\right)\right) \leqq d$. But $\nu(\bar{n}(\bar{V}))=\nu\left(n^{\prime \prime}\left(V^{\prime \prime}\right)\right)$, so that $d \geqq \nu(\bar{n}(\bar{V})) \geqq \nu\left(n^{\prime}\left(V^{\prime}\right)\right)$ $=d$. Since $(16)$ implies that $\omega_{\lambda}^{\prime \prime}\left(V^{\prime \prime}\right) \not \equiv 0(\bmod \mathfrak{p})$, we have shown that $B^{\prime \prime}$ is a block of $\mathfrak{M}$ of defect $d$ having a defect group isomorphic to $\mathfrak{D}$. We shall show that $B_{\tau}^{\prime \prime}$ is in fact $B^{\prime \prime}$.

Let $\chi_{\mu}^{\prime \prime}$ be any character of $\mathfrak{M}$ in $B^{\prime \prime}$. In particular, $\chi_{\mu}^{\prime \prime}$ may be $\chi_{\lambda}^{\prime \prime}$. From the definition of blocks we have

$$
\frac{m}{n^{\prime \prime}\left(G^{\prime \prime}\right)} \frac{\chi_{\mu}^{\prime \prime}\left(G^{\prime \prime}\right)}{x_{\mu}^{\prime \prime}}=\frac{m}{n^{\prime \prime}\left(G^{\prime \prime}\right)} \frac{\chi_{\lambda}^{\prime \prime}\left(G^{\prime \prime}\right)}{x_{\lambda}^{\prime \prime}}(\bmod \mathfrak{p}),
$$

for all elements $G^{\prime \prime}$ in $\mathfrak{M}$. If $G^{\prime \prime}$ is in $\mathfrak{F}$, both sides of the congruence become sth roots of unity, and since $(s, p)=1$, the roots of unity are the same. The representation $\mathfrak{X}_{\mu}^{\prime \prime}$ with character $\chi_{\mu}^{\prime \prime}$ will therefore induce an irreducible $\epsilon$ projective representation $\mathfrak{A}_{\mu}$ of $\overline{\mathfrak{T}}$. Let $\chi_{\mu}^{\prime}$ be the character of the irreducible representation $\mathfrak{A}_{\mu} \times \mathfrak{B}$ of $\mathfrak{T}$. If $\omega_{\mu}^{\prime}$ is the linear character associated to $\chi_{\mu}^{\prime}$, and $G^{\prime}$ is any element in $\mathfrak{T}$, then

$$
\omega_{\mu}^{\prime}\left(G^{\prime}\right)=\frac{t}{n^{\prime}\left(G^{\prime}\right)} \frac{\chi_{\mu}^{\prime}\left(G^{\prime}\right)}{x_{\mu}^{\prime}}=\frac{t}{n^{\prime}\left(G^{\prime}\right)} \frac{\alpha_{\mu}\left(G^{\prime}\right)}{\alpha_{\mu}(1)} \frac{\beta\left(G^{\prime}\right)}{\beta(1)}
$$

or

$$
\omega_{\mu}^{\prime}\left(G^{\prime}\right)=\left[\frac{h}{s} \frac{\beta\left(G^{\prime}\right)}{\beta(1)} \frac{n^{\prime \prime}\left(G^{\prime \prime}\right)}{n^{\prime}\left(G^{\prime}\right)}\right] \frac{m}{n^{\prime \prime}\left(G^{\prime \prime}\right)} \frac{\alpha_{\mu}(\bar{G})}{\alpha_{\mu}(1)},
$$

where $\bar{G}=f\left(G^{\prime}\right)$ and $G^{\prime \prime}=\rho(\bar{G})$. We note the term in the brackets is always a local integer, since $\nu\left(n^{\prime \prime}\left(G^{\prime \prime}\right)\right)=\nu(\bar{n}(\bar{G})) \geqq \nu\left(n^{\prime}\left(G^{\prime}\right)\right)$. But

$$
\frac{m}{n^{\prime \prime}\left(G^{\prime \prime}\right)} \frac{\alpha_{\mu}(\bar{G})}{\alpha_{\mu}(1)} \equiv \frac{m}{n^{\prime \prime}\left(G^{\prime \prime}\right)} \frac{\alpha_{\lambda}(\bar{G})}{\alpha_{\lambda}(1)}(\bmod \mathfrak{p}),
$$

this being obtained from (17) by multiplying by an sth root of unity if necessary. Therefore by (18)

$$
\omega_{\mu}^{\prime}\left(G^{\prime}\right)=\omega_{\lambda}^{\prime}\left(G^{\prime}\right)(\bmod \mathfrak{p})
$$

for all $G^{\prime}$ in $\mathfrak{T}$ and $\chi_{\mu}^{\prime}$ must be in the block $B_{\tau}^{\prime}$.

Let $B^{\prime}$ be the set of all the irreducible characters $\chi_{\mu}^{\prime}$ of $\mathfrak{T}$ obtained from the characters $\chi_{\mu}^{\prime \prime}$ in $B^{\prime \prime}$ in the way just described. We have just shown that $B^{\prime} \subseteq B_{\tau}^{\prime}$. If we can show $B^{\prime}$ is a block of $\mathfrak{T}$, then $B^{\prime}=B_{\tau}^{\prime}$, and the set $B^{\prime \prime}$ is simply $B_{\tau}^{\prime \prime}$. Let $P^{\prime}$ be a $p$-singular element in $\mathfrak{T}, R^{\prime}$ a $p$-regular element in $\mathfrak{T}$. 


$$
\begin{aligned}
\sum_{B^{\prime}} \chi_{\mu}^{\prime}\left(P^{\prime}\right) \chi_{\mu}^{\prime}\left(R^{\prime}\right) & =\beta\left(P^{\prime}\right) \beta\left(R^{\prime}\right) \sum_{B^{\prime}} \alpha_{\mu}\left(P^{\prime}\right) \alpha_{\mu}\left(R^{\prime}\right) \\
& =\beta\left(P^{\prime}\right) \beta\left(R^{\prime}\right) \sum_{B^{\prime \prime}} \chi_{\mu}^{\prime \prime}\left(P^{\prime \prime}\right) \chi_{\mu}^{\prime \prime}\left(R^{\prime \prime}\right),
\end{aligned}
$$

where $P^{\prime \prime}=\rho\left(f\left(P^{\prime}\right)\right), R^{\prime \prime}=\rho\left(f\left(R^{\prime}\right)\right)$. Since $f\left(R^{\prime}\right)$ is $p$-regular, $R^{\prime \prime}$ must be $p$ regular by an earlier remark. If $P^{\prime \prime}$ were also $p$-regular, then $\pi\left(P^{\prime \prime}\right)=f\left(P^{\prime}\right)$ would be $p$-regular, which is not possible. Thus $P^{\prime \prime}$ is $p$-singular, and the sum $\sum_{B^{\prime \prime}} \chi_{\mu}^{\prime \prime}\left(P^{\prime \prime}\right) \chi_{\mu}^{\prime \prime}\left(R^{\prime \prime}\right)=0$. Applying the theorem of Osima as in $(2 \mathrm{~A})$, we see that $B^{\prime}$ is $B_{\tau}^{\prime}$. This completes the proof.

We note that the correspondence $\chi_{\mu}^{\prime} \leftrightarrow \chi_{\mu}^{\prime \prime}$ is height-preserving, since $x_{\mu}^{\prime}=x_{\mu}^{\prime \prime} \times \beta(1)$ and $\nu(m)=\nu(t)$. Consider the restriction of $\beta$ to $\mathfrak{S}$. Since the factor set $\epsilon$ is actually a factor set of $\mathfrak{T} / \mathfrak{W}, \epsilon_{G, H}=1$ whenever both $G$ and $H$ are in $\mathfrak{E}$. Thus $\beta \mid \mathfrak{S}$ is actually $\theta_{\rho}$. Since $\theta_{\rho}$ is an irreducible modular character, it follows a fortiori that as a modular projective representation, $\mathfrak{B}$ must be irreducible. By this we mean that if a suitable basis has been found for which the matrices of $B$ have local integers as coefficients, then the representation $\mathfrak{B}^{*}$ obtained from $\mathfrak{B}$ by the mapping $\mathfrak{D} \rightarrow \Omega^{*}$ is an irreducible modular projective representation. The factor set for $\mathfrak{B}^{*}$ is $\left(\epsilon^{*}\right)^{-1}$, where $\epsilon^{*}$ is the factor set obtained from $\epsilon$ by the mapping $0 \rightarrow \Omega^{*}$. Let

$$
\chi_{\mu}^{\prime \prime}=\sum_{\alpha} d_{\mu \alpha}^{\prime \prime} \phi_{\alpha}^{\prime \prime}
$$

be the modular decomposition of a character $\chi_{\mu}^{\prime \prime}$ in $B_{\tau}^{\prime \prime}$, where the $\phi_{\alpha}^{\prime \prime}$ are the irreducible modular characters of $\mathfrak{M}$. If $\mathfrak{F}_{\alpha}^{\prime \prime}$ is an irreducible modular representation of $\mathfrak{M}$ whose character is $\phi_{\alpha}^{\prime \prime}$, it is easily seen that $\mathfrak{F}_{\alpha}^{\prime \prime}$ induces an irreducible $\epsilon^{*}$ projective representation $\overline{\mathfrak{F}}_{\alpha}$ of $\overline{\mathfrak{T}}$. For the elements $\rho(\bar{G})$, we have $\phi_{\alpha}^{\prime \prime}(\rho(\bar{G}))=\bar{\phi}_{\alpha}(\bar{G})$ for $\bar{G} p$-regular, where $\bar{\phi}_{\alpha}$ is the character of the representation $\bar{\mho}_{\alpha}$. Since the results of Clifford are still valid for the modular case, it follows that $\overline{\mathfrak{F}}_{\alpha} \times \mathfrak{F}^{*}$ is an irreducible modular representation $\mathfrak{F}_{\alpha}^{\prime}$ of $\mathfrak{T}$. Conversely, every irreducible modular representation $\mathfrak{F}_{\alpha}^{\prime}$ of $\mathfrak{T}$ in the block $B_{r}^{\prime}$ can be gotten in this way. As in the ordinary case, $\mathfrak{F}_{\alpha}^{\prime}$ and $\mathfrak{F}_{\beta}^{\prime}$ are equivalent if and only if $\mathfrak{F}_{\alpha}^{\prime \prime}$ and $\mathfrak{F}_{\beta}^{\prime \prime}$ are equivalent. Let $\phi_{\alpha}{ }^{\prime}=\bar{\phi}_{\alpha} \times \beta$ be the character of $\mathfrak{F}_{\alpha}^{\prime}$. If $V^{\prime}$ is a $p$-regular element in $\mathfrak{I}$ and $V^{\prime \prime}=\rho\left(f\left(V^{\prime}\right)\right)$, then $\chi_{\mu}^{\prime}\left(V^{\prime}\right)$ $=\alpha_{\mu}\left(V^{\prime}\right) \beta\left(V^{\prime}\right)=\chi_{\mu}^{\prime \prime}\left(V^{\prime \prime}\right) \beta\left(V^{\prime}\right)$. From (19) we have

$$
\begin{aligned}
\chi_{\mu}^{\prime}\left(V^{\prime}\right) & =\sum_{\alpha} d_{\mu \alpha}^{\prime \prime} \phi_{\alpha}^{\prime \prime}\left(V^{\prime \prime}\right) \beta\left(V^{\prime}\right) \\
& =\sum_{\alpha} d_{\mu \alpha}^{\prime \prime} \Phi_{\alpha}\left(V^{\prime}\right) \beta\left(V^{\prime}\right) \\
& =\sum_{\alpha} d_{\mu \alpha}^{\prime \prime} \phi_{\alpha}^{\prime}\left(V^{\prime}\right) .
\end{aligned}
$$


Since the $\phi_{\alpha}^{\prime}$ are linearly independent, $d_{\mu \alpha}^{\prime}=d_{\mu \alpha}^{\prime \prime}$. In particular, the correspondence $\phi_{\alpha}^{\prime} \leftrightarrow \phi_{\alpha}^{\prime \prime}$ is 1-1 between the modular characters of $B_{r}^{\prime}$ and $B_{r}^{\prime \prime}$. If $c_{\alpha \beta}^{\prime}$ and $c_{\alpha \beta}^{\prime \prime}$ are the Cartan invariants relating $\phi_{\alpha}^{\prime}$ and $\phi_{\beta}^{\prime}, \phi_{\alpha}^{\prime \prime}$ and $\phi_{\beta}^{\prime \prime}$ respectively, then $c_{\alpha \beta}^{\prime}=c_{\alpha \beta}^{\prime \prime}$. Combining these results together with (2B), we have

THEOREM (2D). Let (S) be a group with a nontrivial normal $p^{\prime}$-subgroup $\mathfrak{W}$. Let $B_{r}$ be a block of $(S)$ and suppose the subgroup $\mathfrak{T}$ in (2B) is $B$. Then there is a group $\mathfrak{M}$ and a block $B_{r}^{\prime \prime}$ of $\mathfrak{M}$ such that the following are true: (i) $B_{\tau}$ and $B_{\tau}^{\prime \prime}$ have isomorphic defect groups. (ii) There is a 1-1 height-preserving correspondence $\chi_{\mu}^{\leftrightarrow} \chi_{\mu}^{\prime \prime}$ between the ordinary characters of $B_{\tau}$ and $B_{\tau}^{\prime \prime}$. (iii) There is a 1-1 correspondence $\phi_{\alpha} \leftrightarrow \phi_{\alpha}^{\prime \prime}$ between the modular characters of $B_{\tau}$ and $B_{\tau}^{\prime \prime}$. (iv) With respect to these correspondences of characters, the matrices of decomposition numbers and Cartan invariants of $B_{\tau}$ and $B_{\tau}^{\prime \prime}$ are the same. The group $\mathfrak{M}$ has the following structure: There exists a cyclic normal $p^{\prime}$-subgroup $\mathbb{F}$ in the center of $\mathfrak{M}$ such that $\mathfrak{M} / \mathfrak{F} \cong(\mathfrak{S} / \mathfrak{S}$.

REMARK. It is clear from the construction of $\mathfrak{M}$ that if $\mathfrak{H}$ is $p$-solvable, so is $\mathfrak{M}$, since subgroups and factor groups of $p$-solvable groups are again p-solvable.

3. Arithmetic on $p$-solvable groups. Theorem (2D) leads us to the following reduction: Suppose $B_{\tau}$ is a block of a $p$-solvable group $\$(S$, and we want to prove by induction on the order of $(\$)$ statements on certain quantities associated to $B_{\tau}$. These quantities are (i) the structure of the defect group of $B_{\tau}$, (ii) the heights of the characters in $B_{\tau}$, (iii) the number of ordinary and modular irreducible characters in $B_{r}$, (iv) the decomposition numbers and Cartan invariants of $B_{\tau}$. Then we may assume $B_{\tau}$ is a block of defect $\left.a, a=\nu(B): 1\right)$. For let $\mathfrak{S}$ be the maximal normal $p^{\prime}$-subgroup of $\mathfrak{S}$. If the subgroup $\mathfrak{T}$ of (2B) is not equal to $(B)$, then induction can be used to prove the statements. However, if $\mathfrak{T}=(\mathfrak{S}$, then it follows by (2D) that it will be equivalent to proving the same statements for the block $B_{r}^{\prime \prime}$ of $\mathfrak{M}$ given in (2D). Now by construction $a=\nu(\mathfrak{M}: 1)=\nu(\mathbb{S}: 1)$. Since $\mathfrak{M} / \mathfrak{F} \cong(\mathfrak{S} / \mathfrak{F}$ it follows that $\mathbb{E}$ must be the maximal normal $p^{\prime}$-subgroup of $\mathfrak{M}$. Choose $\mathfrak{R}$ that normal subgroup of $\mathfrak{M}$ such that $\mathfrak{E} \subseteq \mathbb{R} \subseteq \mathfrak{M}$ and $\mathfrak{R} / \mathfrak{F}$ is the maximal normal $p$-subgroup in $\mathfrak{M} / \mathfrak{F}$. By Lemma 1.2 .3 of [8], the centralizer of $\mathscr{R} / \mathfrak{F}$ in $\mathfrak{M} / \mathfrak{F}$ is contained in $\mathscr{R} / \mathfrak{F}$. $\mathfrak{F}$ has order coprime to its index in $\mathbb{R}$ so that by a theorem of Schur, $\mathbb{R}=\mathfrak{D} \mathbb{E}$, where $\mathfrak{D}$ is a $p$-group of order $(\mathbb{R}: \mathfrak{F})$ and $\mathfrak{I} \cap(\xi=1$. Since $\mathfrak{F}$ is contained in the center of $\mathfrak{M}$, we have in fact that $\mathfrak{R}=\mathfrak{D} \times \mathfrak{E}$. $\mathfrak{D}$ is characteristic in $\mathfrak{l}$ and therefore $\mathfrak{D}$ is normal in $\mathfrak{M}$. Let $V$ be a $p$-regular element in $\mathfrak{M}$ such that $V$ centralizes $\mathfrak{D}$. Then $V \&$ centralizes $\mathcal{R} \mathbb{E}$ and consequently $V \mathbb{E} \subseteq \mathbb{R}$ and $V$ belongs to $\mathscr{E}$. If $\mathfrak{B}$ is a defect group of a block of $\mathfrak{M}$, then $\mathfrak{D} \subseteq \mathfrak{P}$ so that $\mathfrak{S}(\mathfrak{P}) \subseteq \mathfrak{S}(\mathfrak{D})$. This means that the only $p$-regular elements of $\mathfrak{M}$ which centralize $\mathfrak{B}$ are the elements of $\mathfrak{F}$, and it then follows that all the blocks of $\mathfrak{M}$ have defect $a$. The block $B_{\tau}$ of (S) must also then have defect $a$. 
LEMma (3A). Let $\$$ be a p-solvable group. Then the following are equivalent:

(i) (S) has only trivial normal $p^{\prime}$-subgroups.

(ii) (S) has only one block, the block $B_{1}$ containing the principal or 1-character of $B$.

Proof. (i) $\Rightarrow$ (ii). Let $\mathfrak{B}$ be the largest normal $p$-subgroup of $\mathfrak{B}$. By Lemma 1.2.3 of [8], we must have $\mathfrak{S}(\mathfrak{P}) \subseteq \mathfrak{P}$. Since $\mathfrak{B} \triangleleft G, \mathfrak{B}$ is contained in the defect groups $\mathfrak{D}$ of every block of $\mathfrak{S}$. In particular, $\mathfrak{S}(\mathfrak{D}) \subseteq \mathfrak{S}(\mathfrak{P}) \subseteq \mathfrak{B}$, and the only $p$-regular element of $B$ which commutes with $\mathfrak{D}$ is the identity element of $\mathbb{S}$. It then follows that $B_{1}$ is the only block of $B$.

(ii) $\Rightarrow$ (i). This implication is actually true for all finite groups (5). Suppose $\mathfrak{G}$ is a nontrivial normal $p^{\prime}$-subgroup of $\mathfrak{B}$. We apply the remarks at the beginning of $\$ 2$ to this case. Without using the fact that $\$$ is a $p^{\prime}$-group, we know the following fact: If $J_{1}$ is the set containing the block $B_{1}$, then the corresponding set $\tilde{\mathfrak{J}}_{1}$ of $\mathfrak{S}$ contains a set of blocks of $\mathfrak{S}$ which are permuted transitively by the elements of $B$. But one of these blocks is $\widetilde{B}_{1}$, the block containing the 1 -character of $\mathfrak{W}$. Thus $\widetilde{B}_{1}$ can only be sent into itself. If $\mathfrak{S}$ is a $p^{\prime}$ group, there are then other blocks of $\mathfrak{S}$ not in $\tilde{\mathfrak{J}}_{1}$, and therefore $\mathfrak{S}$ must have a block different from $B_{1}$, which is a contradiction. This completes the proof.

We will use without explicit mentioning the following well-known facts. Let $\mathfrak{S}$ be a normal subgroup of any finite group $\mathcal{S}$. If $\chi_{\mu}$ is an irreducible character of $(S)$ and $\chi_{\mu} \mid \mathfrak{W}=r_{\mu} \sum_{\alpha-1}^{s} \tilde{\chi}_{\alpha}$, where the $\tilde{\chi}_{\alpha}$ are distinct irreducible characters of $\mathfrak{S}$ associated in $\left(\mathfrak{S}\right.$, then $r_{\mu} s$ divides ((S): $\left.\mathfrak{S}\right)$. This is a direct consequence of Clifford's theorem and Theorem VIIa of [11]. If, furthermore, $(\mathfrak{S}: \mathfrak{W})$ is a prime number $q$, then $r_{\mu}$ is always one, and $s$ is always one or $q$. If $\chi_{\mu} \mid \mathfrak{S}$ is reducible, $\chi_{\mu}$ then vanishes for elements of $\mathfrak{S}$ not in $\mathfrak{E}$.

Theorem (3E) connecting the heights of the characters in a block $B_{\tau}$ of $(B)$ to the structure of the defect group of $B_{\tau}$ will be proved in a sequence of statements.

LEMMA (3B). Let $(S$ be any finite group, and $\mathfrak{S}$ a normal subgroup of index $(\mathfrak{S}: \mathfrak{S})=p$. Let $B_{\tau}$ be a block of $\mathbb{H}$ of defect $d$ and defect group $\mathfrak{D}$. If $\chi_{\mu}$ is an irreducible character in $B_{\tau}$ such that $\chi_{\mu} \mid \mathfrak{S}$ is reducible, then the center $\mathbb{B}$ of $\mathfrak{D}$ lies in $\mathfrak{S}$.

Proof. We first note that the character $\chi_{\mu}$ vanishes on elements of $(B)$ not in $\mathfrak{S}$. Let $V$ be a $p$-regular element of $\mathfrak{S}$ such that $\mathfrak{D}$ is a defect group of $V$ in $\mathfrak{G}$, and such that $\omega_{\mu}(V) \not \equiv 0(\bmod \mathfrak{p})$. Since $(\mathfrak{S}: \mathfrak{S})=p, V$ is actually in $\mathfrak{S}$. Suppose $A$ is an element in $\mathbb{Z}$ but not in $\mathfrak{S}$. Then $A V$ is not in $\mathfrak{S}$ and consequently $\chi_{\mu}(A V)=0, \omega_{\mu}(A V)=0$. Let $\chi_{\lambda}$ be a character of height 0 in $B_{\tau}$. Then

$$
\omega_{\lambda}(V) \not \equiv 0, \quad \omega_{\lambda}(A V) \equiv 0(\bmod \mathfrak{p}) .
$$

If we can show that $\nu(A V)=d$, then these congruences imply

$$
\chi_{\lambda}(V) \not \equiv 0 \quad \chi_{\lambda}(A V) \equiv 0(\bmod \mathfrak{p})
$$


This would be impossible, for since $A$ and $V$ commute and $A$ has order a $p$-power, $\chi_{\lambda}(A V) \equiv \chi_{\lambda}(V)(\bmod \mathfrak{p})$. To show $\nu(A V)=d$, we note that $\mathfrak{D} \subseteq \mathfrak{R}(A)$, $\mathfrak{D} \subseteq \mathfrak{N}(V)$. Thus $\mathfrak{D} \subseteq \mathfrak{N}(A V)$ and $\nu(A V) \geqq d$. But from $\mathfrak{N}(A V)=\mathfrak{N}(A) \cap \mathfrak{N}(V)$, $n(A V)$ must be a divisor of $n(V)$ so that in fact, $\nu(A V)=d$.

TheOREM (3C). Let $\$$ be a finite p-solvable group, and let $B_{\tau}$ be a block of (S) of defect $d$ and defect group $\mathfrak{D}$. If the center $\mathfrak{B}$ of $\mathfrak{D}$ has index $p^{c}$ in $\mathfrak{D}$, then every character in $B_{\tau}$ has height not exceeding $c$.

Proof. The proof is by induction on the order $g$ of $(S)$, the theorem being trivial for either a $p^{\prime}$-group or a $p$-group of prime order. By the remarks at the beginning of $\$ 3$ and $(3 \mathrm{~A})$, we may assume that $B_{\tau}$ has defect $a$. In this case, the defect group $\mathfrak{D}$ is a Sylow $p$-subgroup of $B$. Let $\chi_{\mu}$ be a character in $B_{\tau}$ of degree $x_{\mu}$. Then $\nu\left(x_{\mu}\right)=(a-a)+e_{\mu}=e_{\mu}$, where $\nu(g)=a$ and $e_{\mu}$ is the height of $\chi_{\mu}$. Let $\mathfrak{S}$ be a maximal normal subgroup of $(\mathfrak{S}$. The constituents $\tilde{\chi}_{\alpha}$ of $\chi_{\mu} \mid \mathfrak{S}$ belong to blocks of $\mathfrak{S}$ of defect $a$ (see $(1 \mathrm{~F})$ ). If (G): $\left.\mathfrak{Q}\right)$ is prime to $p$, then $\mathfrak{D}$ is also a Sylow $p$-subgroup of $\mathfrak{T}$ and $\nu\left(x_{\mu}\right)=\nu\left(\tilde{x}_{\alpha}\right)=e_{\mu} \leqq c$ by the induction hypothesis. Suppose then that $(\mathfrak{S}: \mathfrak{E})=p$. $\tilde{\mathfrak{D}}=\mathfrak{D} \cap \mathfrak{F}$ is a defect group for $\widetilde{B}_{1}$ and $\mathfrak{Z} \cap \mathfrak{S} \subseteq \tilde{\mathbb{B}}$, where $\tilde{\mathbb{B}}$ is the center of $\tilde{\mathfrak{D}}$. If $\chi_{\mu} \mid \mathfrak{S}$ is irreducible, then $\nu\left(x_{\mu}\right)=e_{\mu} \leqq \nu(\tilde{\mathfrak{D}}: \tilde{\mathfrak{X}}) \leqq \nu(\mathfrak{D}: \mathfrak{Z})=c$. If $\chi_{\mu} \mid \mathfrak{W}$ is reducible and $\tilde{\chi}_{\alpha}$ is an irreducible constituent of $\chi_{\mu} \mid \mathfrak{S}$, then the degrees $\tilde{x}_{\alpha}$ of $\tilde{\chi}_{\alpha}$ and $x_{\mu}$ of $\chi_{\mu}$ are related by $x_{\mu}=p \tilde{x}_{\alpha}$. The height of $\tilde{\chi}_{\alpha}$ is then $e_{\mu}-1$. By (3B) $3 \subseteq \tilde{B}$ so that by the induction hypothesis, $e_{\mu}-1 \leqq \nu(\tilde{D}: \tilde{\mathfrak{Z}}) \leqq \nu(\tilde{D}: \mathfrak{Z})=c-1$, and $e_{\mu} \leqq c$.

LEMMA (3D). Let $\$$ be a finite p-solvable group of order $g$. If every irreducible character of (\$) has degree prime to $p$, then (S) has a normal abelian Sylow $p$ subgroup.

Proof. We proceed by induction on $g$, the lemma being trivial for either a $p$-group or a $p^{\prime}$-group. Let $\mathfrak{S}$ be a maximal normal subgroup of $\mathbb{G}$. Every irreducible character of $\mathfrak{S}$ also has degree prime to $p$, so that by the induction hypothesis, $\mathfrak{S}$ has a normal abelian Sylow $p$-subgroup $\tilde{\mathfrak{P}}$. Since $\tilde{\mathfrak{P}}$ is a characteristic subgroup of $\mathfrak{S}, \tilde{\mathfrak{B}} \triangleleft \mathfrak{S}$. Thus if $p \nmid(\mathfrak{S}: \mathfrak{S}), \tilde{\mathfrak{P}}$ is even a Sylow subgroup of $\$ 5$. We may then assume that $(\mathfrak{S}: \mathfrak{S})=p$. Suppose first that $p^{2} \mid g$; the factor group $\$ / \tilde{\mathfrak{B}}$ is then of order $<g$. Its irreducible characters, identified as characters of $\mathbb{S}$, have degree prime to $p$, and therefore its Sylow $p$-subgroup $\mathfrak{B} / \tilde{\mathfrak{P}}$ is then normal in $\mathbb{S} / \tilde{\mathfrak{B}}$. The subgroup $\mathfrak{P}$ of $\mathbb{S}$ corresponding to $\mathfrak{B} / \tilde{\mathfrak{P}}$ is then a normal Sylow $p$-subgroup of $\mathfrak{B}, \mathfrak{P}$ having an abelian normal subgroup $\tilde{\mathfrak{P}}$ of index $p$. Now the irreducible characters of $\mathfrak{S}$ are all left fixed by the inner automorphisms of $\mathfrak{B}$. By applying $(1 \mathrm{~A})$ to the 1-block of $\mathfrak{F}$, we see that for any element $P$ in $\tilde{\mathfrak{B}}, n(P)=p \tilde{n}(P)$, where $\tilde{n}$ is the order of the normalizer $\tilde{\mathfrak{N}}$ in $\mathfrak{S}$. Since $\tilde{\mathfrak{B}}$ is abelian, $\tilde{\mathfrak{P}} \subseteq \tilde{\mathfrak{N}}(P)$. $\mathfrak{N}(P)$ then contains a Sylow $p$-subgroup of $\mathfrak{S}$ which is necessarily $\mathfrak{P}$. Thus $\tilde{\mathfrak{P}}$ is contained in the center of $\mathfrak{P}$ and $\mathfrak{P}$ is abelian. 
Finally the case $p^{2} \nmid g$. Let $P$ be an element of order $p$ and $\mathfrak{P}$ the group generated by $P$ in $(5)$. $P$ induces an automorphism $\alpha$ of $\mathfrak{S}$ by transformation. To show that $\mathfrak{B} \triangleleft \mathfrak{G}$, we need only show that $\alpha$ is the identity automorphism of $/ \mathfrak{S}$. The assumption on the degrees of the characters of $\mathbb{B}$ implies that $\alpha$ maps each conjugate class of $\mathfrak{S}$ onto itself. Since $p \nmid(\mathfrak{E}: 1)$, it follows from Satz 108 of [12] that $\alpha$ is indeed the identity.

Theorem (3E). Let $(S)$ be a finite p-solvable group and let $B_{r}$ be the block containing the 1-character. Then a Sylow p-subgroup $\mathfrak{D}$ is abelian if and only if every character $\chi_{\mu}$ in $B_{\tau}$ has height $O$.

Proof. The only if part follows from (3C). We prove the if part by induction. For a $p$-group or a $p^{\prime}$-group, the theorem is trivial. Let $\mathfrak{F}$ be the maximal normal $p^{\prime}$-subgroup of $(3)$. The proof of $(3 \mathrm{~A})$ shows that every character of $(S) / S$, when considered as a character of $B$, belongs to the 1 -block $B_{\tau}$ of $\mathbb{S}$. By (3D) the Sylow $p$-subgroups of $(5) / \mathfrak{S}$ are therefore abelian, and $\mathfrak{D}$ must be abelian. This completes the proof.

The height of an irreducible modular character of any finite group \& can be defined as for the ordinary irreducible characters of $(S)$. If $\phi_{\rho}$ is an irreducible modular character of $B$ in a block of defect $d$ and $\nu(\$): 1)=a$, then the height of $\phi_{\rho}$ is the non-negative integer $h_{\rho}$, where $\nu\left(\phi_{\rho}(1)\right)=a-d+h_{\rho}$. It is easily seen that the correspondence $\phi_{\rho} \leftrightarrow \phi_{\rho}^{\prime \prime}$ in (2D) is height-preserving. For the modular characters, we shall prove an analogue of $(3 \mathrm{C})$, but as the example of $p$ groups shows, no analogue to $(3 \mathrm{E})$ exists.

THeOREM (3F). Let (S) be a finite p-solvable group, and let $B_{\tau}$ be a block of (5) of defect $d$ and defect group $\mathfrak{D}$. If the center $\mathfrak{B}$ of $\mathfrak{D}$ has index $p^{c}$ in $\mathfrak{D}$, then every modular character in $B_{\tau}$ has height not exceeding $c$.

Proof. The proof is by induction on the order $g$ of $B$, the theorem being trivial for either a $p^{\prime}$-group or a $p$-group of prime order. By the reduction principle we may assume that $B_{\tau}$ is a block of defect $a$. In this case the defect group $\mathfrak{D}$ is a Sylow $p$-subgroup of $\left(5\right.$. Let $\phi_{\rho}$ be a modular character in $B_{\tau}$; the height $h_{\rho}$ of $\phi_{\rho}$ is then $\nu\left(\phi_{\rho}(1)\right)$. Let $\mathfrak{S}$ be a maximal normal subgroup of $\mathbb{S}$. If $p \nmid(\mathfrak{S}: \mathfrak{S})$ and $\tilde{\phi}_{\rho}$ is an irreducible constituent of $\phi_{\rho} \mid \mathfrak{S}$, then $\nu\left(\tilde{\phi}_{\rho}(1)\right)=h_{\rho} \leqq c$ by the induction hypothesis $\left({ }^{3}\right)$. We may suppose then that $(\mathbb{S}: \mathfrak{S})=p$. $\tilde{\mathfrak{D}}=\mathfrak{D} \cap \mathfrak{S}$ is a Sylow $p$-subgroup of $\mathfrak{S}$ and $\mathfrak{Z} \cap \mathfrak{S} \subseteq \tilde{\mathbb{Z}}$, where $\tilde{\mathbb{Z}}$ is the center of $\tilde{\mathfrak{D}}$. If $\phi_{\rho} \mid \mathfrak{S}$ is irreducible, then $\nu\left(\phi_{\rho}(1)\right)=h_{\rho} \leqq \nu(\tilde{\mathfrak{D}}: \tilde{\mathfrak{Z}}) \leqq \nu(\mathfrak{D}: \mathbb{Z})=c$. We may finally suppose that $\phi_{\rho} \mid \mathfrak{S}$ is reducible, say that $\phi_{\rho} \mid \mathfrak{S}=\tilde{\phi}_{\rho_{1}}+\tilde{\phi}_{\rho_{2}}+\cdots+\tilde{\phi}_{\rho_{p}}$, the height of the $\tilde{\phi}_{\rho_{i}}$ being $h_{\rho}-1$. If some irreducible character $\chi_{\mu}$ of $B$ is reducible as a character of $\mathfrak{S}$, then by $(3 \mathrm{~B}) \quad 3 \subseteq \tilde{\mathbb{B}}$ so that $h_{\rho}-1 \leqq \nu(\tilde{\mathfrak{D}}: \tilde{\mathfrak{Z}})$ $\leqq \nu(\tilde{\mathfrak{D}}: \mathfrak{3})=c-1$, and $h_{\rho} \leqq c$. But if every character $\chi_{\mu}$ in $B_{\tau}$ were irreducible

(3) If $\tilde{\phi}_{\rho}$ occurs with multiplicity $s$ in the restriction of $\phi_{\rho}$ to $\mathfrak{E}$, then $s$ is the degree of an irreducible modular projective representation of some subgroup of $\mathbb{S} / \mathfrak{W}$. But since $\mathbb{S} / \mathfrak{W}$ is a $p^{\prime}$-group, the classical and modular theories agree, and by Satz VIIa of [11], $s$ divides ((S: $\left.\mathfrak{E}\right)$. 
as a character of $\mathfrak{S}$, then the columns of decomposition numbers of $\mathfrak{S}$ corresponding to the modular characters $\tilde{\phi}_{\rho_{i}}$ would be linearly dependent, which is impossible by $[2,(5 \mathrm{~A})]$.

Corollary (3G). The degrees of the irreducible modular characters $\phi_{p}$ of a $p$-solvable group (S) of order $g$ satisfy the relation $\nu\left(\phi_{p}(1)\right) \leqq \nu(g)$.

THEOREM (3H). Let (s) be a p-solvable group and let $B_{r}$ be a block of defect $d$. The Cartan invariants $c_{\rho \sigma}$ of $B_{\tau}$ satisfy the inequality $c_{\rho \sigma} \leqq p^{d}$.

Proof. The proof being by induction, we may assume that $B_{r}$ is a block of defect $a$. Since $B$ is $p$-solvable, there exists a subgroup $\mathfrak{E}$ of $B$ of index $p^{a}$, where $\nu(B: 1)=a$ (see [7]). But by Theorems 8 and 9 of [5], $c_{\rho \sigma} \leqq p^{a}$.

The final theorem is one for solvable groups. Its proof will require a more detailed look at the case of a normal subgroup $\mathfrak{S}$ of prime index in $\mathbb{S}$. The notation will be that of $\$ 1$.

Lemma (3J). Let $\mathfrak{S}$ be a normal subgroup of the group (s) of prime index $q$. If $B_{\tau}$ is a block of $B$ in the set $\Im_{i}$, then $B_{r}$ is a block of maximal defect in $\mathfrak{J}_{i}$.

Proof. If $\chi_{\lambda}$ is an irreducible character of $\mathbb{B}$ such that $\chi_{\lambda} \mid \mathfrak{S}$ is irreducible, we shall say that the $q$ irreducible characters of $\mathbb{B}$ whose restrictions to $\mathfrak{E}$ are equal to $\chi_{\lambda} \mid \mathfrak{W}$ are associated. These $q$ characters are equal on elements of $\mathfrak{W}$, and for an element $G$ not in $\mathfrak{S}$, their values on $G$ are gotten by multiplying $\chi_{\lambda}(G)$ by the $q$ different roots of unity. Suppose $\chi_{\mu}$ is a character in $B_{r}$ such that $\chi_{\mu} \mid \mathfrak{W}$ is reducible. If $\chi_{\lambda}$ is any other character in $B_{\tau}$, then

$$
\omega_{\lambda}(G) \equiv \omega_{\mu}(G)=0(\bmod \mathfrak{p})
$$

for $G$ not in $\mathfrak{S}$. In particular, if $\chi_{\lambda} \mid \mathfrak{S}$ is irreducible, (20) implies that the associates of $\chi_{\lambda}$ are also in the block $B_{r}$. Thus it is impossible to construct a chain of characters of $B$ satisfying the conditions of (1C) which involves characters not in $B_{\tau}$, and $B_{\tau}$ is the only block of $B$ in in $J_{i}$.

We may then assume that the restriction to $\mathfrak{S}$ of every character $\chi_{\mu}$ in $B_{\tau}$ is an irreducible character $\tilde{\chi}_{\mu}$. Let these be indexed by the set $S$. We shall show that the set $\widetilde{B}$ of characters $\tilde{\chi}_{\mu}, \mu$ in $S$, is a block of $\mathfrak{G}$, and at the same time, that either $B_{r}$ is the only block in $\Im_{i}$, or there are $q$ blocks in $\Im_{i}$, each block containing one character from the family of $q$ characters associated to $\chi_{\mu}, \mu$ in $S$. In either case, $B_{\tau}$ will be of maximal defect in $\mathfrak{J}_{i}$. Let $V$ be a $p$ regular element in $\mathfrak{S}, R$ a $p$-singular element in $\mathfrak{G}$. By [3, (7C) ],

$$
\sum_{\mu \text { in } S} \chi_{\mu}(R) \chi_{\mu}(V)=0 .
$$

Suppose $q=p$. All the $p$-regular elements of $G$ are contained in $\mathfrak{S}$ and thus the associates of $\chi_{\mu}$, for $\mu$ in $S$, are also in $B_{r}$. In this case (21) can be rewritten, after factoring out the constant $p$, as 


$$
\sum_{\mu \text { in } S} \tilde{\chi}_{\mu}(R) \tilde{\chi}_{\mu}(V)=0
$$

and the theorem of Osima implies that $\widetilde{B}$ is a union of blocks of $\mathfrak{S}$ and hence a block of $\mathfrak{S}$. Suppose $q \neq p$. If $\psi_{\tau}(G)=0$ for all $p$-regular elements $G$ not in $\mathfrak{W}$, the associates of a character $\chi_{\mu}$ in $B_{\tau}$ will also be in $B_{\tau}$ and the same argument as in the case $q=p$ can be applied. Suppose finally that $X$ is a $p$-regular element not in $\mathfrak{S}$ such that $\psi_{\tau}(X) \neq 0$. If $\chi_{\mu}$ is an irreducible character in $B_{r}$, the values on $X$ of the linear characters determined by the associates of $\chi_{\mu}$ are the $q$ different products of $\omega_{\mu}(X)$ by $q$ th roots of unity. Since the mapping $0 \rightarrow \Omega^{*}$ distinguishes $q$ th roots of unity, $B_{\tau}$ 'contains no associates of $\chi_{\mu}$ besides $\chi_{\mu}$ itself. Again (22) holds, this time being the same equation as (21), and the same argument can be applied.

THEOREM (3K). Let $\$$ be a finite solvable group of order $g=\prod p_{i}^{a_{i}}$, where the $p_{i}$ are the distinct prime divisors of $g$. Let $\chi_{\mu}$ be an irreducible character of $(S)$ belonging for each prime $p_{i}$ to a block whose defect group $\mathfrak{D}_{i}$ has exponent $p_{i}{ }^{{ }^{i}}$. Then the representation whose character is $\chi_{\mu}$ can be written in the field $\Delta$ of nth roots of unity, where $n=\prod p_{i}{ }^{{ }^{i}}$.

Proof. The theorem is trivial for prime order groups; we proceed by induction. Let $\mathfrak{S}$ be a maximal normal subgroup of $\mathfrak{B}$. By (3J) and (1F) an irreducible constituent $\tilde{\chi}_{\mu}$ of $\chi_{\mu} \mid \mathfrak{S}$ belongs for each prime $p_{i}$ to a block of $\mathfrak{S}$ whose defect group is isomorphic to a subgroup of $\mathfrak{D}_{i}$. By induction the representation whose character is $\tilde{\chi}_{\mu}$ can be written in $\Delta$. An element $G$ of $B$ can be expressed in the form $G=\prod P_{i}$, where the $P_{i}$ are powers of $G$ such that the order of $P_{i}$ is a power of $p_{i}$. The character $\chi_{\mu}$ is zero on $G$ if for some $i, P_{i}$ is not conjugate to some element of $\mathfrak{D}_{i}$ (see $[3,(7 \mathrm{~A})]$ ). Thus if $\chi_{\mu}(G) \neq 0$, then $G$ has order dividing $n$. In particular, it follows that the values of $\chi_{\mu}$ are in $\Delta$. Let $m$ be the Schur index of $\chi_{\mu}$ with respect to $\Delta ; m$ is the smallest positive integer such that $m \chi_{\mu}$ is the character of an irreducible representation of $\&$ over the field $\Delta\left(\chi_{\mu}\right)$ generated by $\Delta$ and the values of $\chi_{\mu}$. In this case $\Delta\left(\chi_{\mu}\right)=\Delta$. Since $\chi_{\mu}$ occurs with multiplicity one in the character of $B$ induced by $\tilde{\chi}_{\mu}, m=1$ by Satz IXa of [10].

\section{REFERENCES}

1. R. Brauer, On the representations of a group of order $g$ in the field of gth roots of unity, Amer. J. Math. vol. 67 (1945) pp. 461-471.

2. - Zur Darstellungstheorie der Gruppen endlicher Ordnung. I, Math. Z. vol. 63 (1956) pp. 406-444.

3. - Zur Darstellungstheorie der Gruppen endlicher Ordnung. II, Math. Z. vol. 72 (1959) pp. 25-46.

4. - Number theoretical investigations on groups of finite order, Proceedings of the International Symposium on Algebraic Number Theory, Tokyo, 1956, pp. 55-62.

5. R. Brauer and C. Nesbitt, On the modular characters of groups, Ann. of Math. vol. 42 (1941) pp. 556-590. 
6. A. H. Clifford, Representations induced in an invariant subgroup, Ann. of Math. vol. 38 (1937) pp. 533-550.

7. S. A. Cunikhin, II-separable groups, Dokl. Akad. Nauk SSSR vol. 59 (1948) pp. 443445.

8. P. Hall and G. Higman, On the p-length of p-soluble groups, Proc. London Math. Soc. ser. 3, vol. 6 (1956) pp. 1-42.

9. M. Osima, Notes on blocks of group characters, Math. J. Okayama Univ. vol. 4 (1955) pp. 175-188.

10. I. Schur, Arithmetische Untersuchungen über endlicher Gruppen linearer Substitutionen, Preuss. Akad. Wiss. Sitzungsber. (1906) pp. 164-184.

11. - Über die Darstellung der endlicher Gruppen durch gebrochene lineare Substitutionen, J. Reine Angew. Math. vol. 127 (1904) pp. 20-50.

12. A. Speiser, Die Theorie der Gruppen von endlicher Ordnung, 3rd ed., Berlin, Springer, 1937.

Cambridge University,

Cambridge, England 